\title{
Alexander Schink Elemente symbolischer Umweltpolitik im Abfallrecht
}

\begin{abstract}
Der Autor geht von der These aus, daß es im Umwelsschutz nicht immer darum geht, tatsächliche Probleme zu bewältigen. Nicht selten wolle der Staat vielmehr mit der Umweltgesetzgebung seine Handlungsfähigkeit und Sensibilitat im Umgang mit den Sorgen von Bürgern demonstrieren. Von dieser These ausgehend untersucht er die Abfallpolitik auf Elemente symbolischer Politik. Er kommt zu dem Ergebnis, daß eine Gesamtkonzeption der Vermeidung von Abfällen und der Produktverantwortung weitgebend fehlt und deshalb die Abfallgesetzgebung eber symbolischen Charkater hat.
\end{abstract}

Die Red.

\section{Symbolische Umweltpolitik als Handlungsmuster des Gesetzgebers}

Umweltschutz hat heute immer noch einen hohen politischen Stellenwert. Folge davon ist, daß Politik, Gesetzgeber und Regierung auf aktuelle Umweltprobleme mit immer neuen Vorschriften reagieren. Festzustellen ist, daß das Umweltreche trotz oder gerade wegen der Beschleunigungsmentalität des Gesetzgebers, die in den letzten Jahren das umweltpolitische Handeln bestimmt hat, umweltrechrliche Vorschriften in immer kürzeren Zeiträumen ändert, verfeinert und kompliziert. Dabei geht es freilich nicht immer darum, Sachprobleme tatsächlich zu bewältigen. Nicht selten kommt es dem Gesetz- und Verordnungsgeber nicht so sehr auf die tatsächliche Durchsetzung von Rechtssätzen, sondern darauf an, zu demonstrieren, daß der Staat handlungsfähig und in der Lage ist, sich der Probleme und Sorgen, der Erwartungen und Hoffnungen der Bürger anzunehmen. Der sozialethische Wert des Umwcltschutzes wird so zwar in Gesetzen und Rechtsverordnungen manifestiert; für die Öffentlichkeit wird Aktionismus initiiert. Dies geschieht jedoch in dem klaren Bewußtsein, daß die neuen Vorschriften rechtlich nicht vollzogen werden können. Ǔmweltrecht bleibt mit anderen Worten häufig im bloß symbolhaften Aktionismus stecken'. Beispiele dafür gibe es zuhauf. Erinnert sei nur an die Staatszielbestimmung Umweltschutz in Art. $20 a \mathrm{GG}$, deren Funktion in erster Linie darin besteht, im Wege eincs Appells zu verdeutlichen, daß Umweltschutz als Staatsziel von allen Staatsebenen und staatlichen Akteuren, also vom Gesetzgeber, der Verwaltung und der Justiz als Daueraufgabe zu begreifen und Umwelterfordernissen ständig Rechnung zu tragen ist. Zur Lösung konkreter Probleme trägt diese Norm indessen nichts oder allenfalls wenig bei². Bloße Symbolik stellt auch das Ozon-

I Zur symbolischen Geserzgebung im Umweltrecht Schink, Vollzugsdefizite im kommunalen Umweltschutz, ZUR 1993, I, sf. Zur symbolisuthen Geseczgebung allgemein Hegenbarth, Symbolische und instrumentelle Funktion moderner Giserze, ZRP 1981, 201 ff; Schmehl, Symbolische Gesetzgebung, ZPR 1991, 25Iff.

2 Dazu Schink, ZUR 1993 (Fn. 1), s. Zur Bedeutung der Staatszielbestimmung Umweltschuty: Hennecke, Der Schutz der naturlichen Lebensgrundlagen in Art. 20a GG, NuR I995, 323 ff.; Kloepfer, Umweltschutz als Verfassungsrecht: Zum neuen Art. $20 \mathrm{a}$ GG, DVBl 1996, 73 ff.; ders., Umweltreche, 2. Aufl. 1998, \$3, Rdnrn. 20ff.; Murswiek, Staatsziel Umweltschutz, NVwZ 1996, 222 ff.; Peters, Art. 20a GG - Die neuc Staatsziclbestimnung des Grundgesetzes, NVwZ 1995, sss ff.; Schink, Umweltschutz als Staatsziel, DOV 
Gesetz ${ }^{3}$ dar, dessen Instrument - Verkehrsbeschränkungen bei Sommersmog - schon vom naturwissenschaftlichen Ansatz her kaum etwas zur Bewältigung des Sommersmogs beiträgt, das zudem die Grenzwerte so hoch setzt, daß mit ihrer Anwendung kaum zu rechnen ist, und dessen vielfältige Ausnahmen dafür Sorge tragen, daß im Ernstfall eigentlich niemand betroffen ist, weil doch (fast) jeder sein Auto benutzen kann.4. Freilich: Symbolische Gesetzgebung muß nicht stets bloßer Aktionismus sein. Ihr Sinn kann auch sein, Ziele zu setzen, Probleme zu verdeutlichen und Wege und Methoden anzugeben, durch die Umweltprobleme gelöst werden können. Solch positive Symbolik serzt auf autonomes Handeln der gesellschaftlichen Kräfte und will staatliches Handeln nur dort ins Spiel bringen, wo gesellschaftliche Selbstregulierungsmechanismen zur Problemlösung ungeeignet sind oder wo die gesellschaftlichen Akteure die umweltpolitischen Ziele des Gesetzgebers nicht oder nicht im notwendigen Umfang aufgreifen. Im folgenden soll der Frage näher nachgegangen werden, inwieweit im Abfallrecht Elemente symbolischer Politik vorkommen.

\section{Das KrW-/AbfG - Neue Zielsetzungen in der Abfallpolitik}

Am 7.6. 1996 ist das $\mathrm{KrW}-/ \mathrm{AbfG}^{5}$ in Kraft getreten ${ }^{6}$. Durch das neue Recht beabsichtigte der Gesetzgeber eine grundsätzliche Neuorientierung in den abfallwirtschaftlichen und abfallrechtlichen Zielsetzungen. Dabei sind vor allem drei Bereiche hervorzuheben:

- Absicht des Gesetzgebers war es, das Recht der Abfallbeseirigung zu einem Recht der Kreislaufwirtschaft fortzuentwickeln. Das bisherige Abfallreche regelte nahezu ausschließlich Fragen der umweltgerechten Entsorgung von Abfällen durch ihre Verwertung oder Beseitigung. Demgegenüber zielt das $\mathrm{KrW}$-/AbfG nicht allein darauf $a b$, sondern versucht durch Regelungen über die Produktverantwortung ( $\mathbb{S} 22 \mathrm{ff} . \mathrm{KrW}-/ \mathrm{AbfG}$ ) bereits auf die Produktion mit dem Ziel einzuwirken, die Entsorgung der späteren $»$ Produktabfällı $«$ mit zu berücksichtigen. Produkte sollen so konstruiert, hergestellt und vertrieben werden, daß von vornherein cine umweltverträgliche Verwertung und Beseitigung sichergestellt ist. Es soll in den Betrieben und bei der Produkrion ein Denken vom Abfall her Einzug halten. Dort soll angestrebt werden die Entwicklung von mehrfach verwendbaren, technisch langlebigen sowie problemlos zu verwertenden und zu beseitigenden Produkten. Der produzierenden Wirtschaft soll damit eine eigene abfallwirtschaftliche Verantwortung auferlegt werden. Im Sinne des Verursacherprinzips soll die private Vermeidungs- und Entsorgungsverantwortung schon bei der Produktion von Stoffen ansetzen, damit insgesamt weniger Abfälle und insbesondere weniger schädliche Abfälle anfallen. Die privaten Produzenten sollen in die Verantwortung genommen werden für den Gedanken der Kreislaufwirtschaft. Abfall, so das neue

1997, $22 \mathrm{ff}$; Steinberg, Verfassungsrechtlicher Umweltschutz durch Grundrechre und Staatszielbcstimnungen, NJW 1996, 1985 ff.; Waechter, Umweltschucz als Staatsziel, NuR 1996, 32 iff.

3 Gescť zur Anderung des Bundesimmissionsschurzgesetzes vom 19. 7. 1999, BGBl. I, 930 ff.

4 Zum Inhalt des Ozon-Gesetzes Schmehl/Karthaus, Die Verkehrsbeschränkungen bci Ozonsmog nach $\$ \$ 401-4$ ce BImSchG, NVwZ 1995, 117 Iff; Schink, Die Entwicklung des Umweltrechts im Jahre I995 Zwerter Teil, ZAU 1996, s2off.; Spechu/Strauß, Ziel und Vollzug des neuen Ozon-Geserzcs, UPR 1995, $38 \mathrm{ff}$.

S Gesetz zur Förderung der Kreislaufwi:tschatt und Sicherung der umweltverträglıchen Beseitigung von Abfallen (Kreislaufwirtschafts- und Abfallgesetz - KrW-/AbtG) v. 27.9. 1994, verkundet als Art. I des Gesetzes zur Vermeidung, Verwertung und Be'veitigung von Abfällen vom 27.9.1994 (BGBI. I, 2705).

$6 \mathrm{~V}_{\mathrm{g}}$. Arr. 13 des Gesetzes zur Vermeidung, Vorwertung und Beseitityng von Abfallon, ebd. (Fn. s). 
Prinzip, soll nicht erst bei seinem Anfall in den Blick geraten, sondern durch vorausschauendes Denken bei der Produktion vermieden, minimiert und in seinen Schadstoffbestandteilen reduziert werden.

- Das KrW-/AbfG zielt weiter darauf ab, eine Kreislaufwirtschaft zu initiieren. Nicht mehr allein die Sicherheit der Beseitigung und ihre Umweltverträglichkeit sind Ziele des Abfallrechts. Aus der bisherigen "Einweg«-Entsorgung zur Verbrennungsanlage oder Deponie soll eine Kreislaufwirtschaft werden ${ }^{7}$. Das Abfallrecht setzt nicht mehr allein am Schadstoffgehalt der zu verwertenden oder beseirigenden Abfälle an, sondern sucht eine ressourcenökomische und ökologische stoffbezogene bzw. materialwirtschaftlich orientierte Kreislaufwirtschaft ins Werk zu setzen ${ }^{8}$. Der Umsetzung dicses Konzeptes dient vor allem die Verankerung einer neuen abfallwirtschaftlichen Zielhierarchie in $\$ 4 \mathrm{Abs}$. I $\mathrm{KrW}-/ \mathrm{AbfG}$ : In einer Art ökologischen Rangfolge ist dort bestimmt, daß Abfälle »in erster Linie zu vermeiden und in zweiter Linie stofflich oder energetisch zu verwerten sind «. Dabei soll bei mehreren Verwertungsoptionen die besser umweltverträgliche Verwertungsart vorrangig sein (vgl. $\$ 6$ Abs. I Satz $2 \mathrm{KrW}-/ \mathrm{AbfG}$ ). Die Beseitigung ist damit gegenüber der Verwertung und jene wiederum gegenüber der Vermeidung nachrangige Entsorgungsart. Im Verhältnis zur Verwertung kommt der Beseitigung nur dann ein Vorrang zu, wenn die Beseitigung des Abfalls »die umweltverträglichere Lösung darstellt« (vgl. $₫ s$ Abs. s KrW-/AbfG).

- In engem Zusammenhang damit steht eine Verschiebung der Entsorgungsverantwortung weg von der ausschließlichen öffentlichen Daseinsvorsorge hin zu mehr privater Verantwortung. $\int S 5$ Abs. 2 und is Abs. I KrW-/AbfG nehmen Abfallerzeuger und -besitzer selbst in die Verantwortung und weisen ihnen nach Maßgabe des Verursacherprinzips die Aufgabe der Verwertung und Bescitigung von Abfällen als eigene zu. Ergänzt wird dieser Paradigmenwechsel' durch weitgehende Möglichkeiten der Übertragung der verbliebenen Entsorgungsverpflichtungen der öffentlichen Hand auf private Dritte ( $\$ 16$ Abs. 2) und Verbände und Einrichtungen der Selbstverwaltungskörperschaften der Wirtschafr (\$S 17, i $8 \mathrm{KrW}-/ \mathrm{AbfG}$ ). Die öffentlich-rechtlichen Gebietskörperschaften sollen lediglich dort tätig werden, wo das Prinzip der privaten Verantwortung für die Abfallverwertung und -beseitigung nicht zu sachgerechten und umweltverträglichen Ergebnissen führt. Insbesondere die Abfallbeseitigung sowie die Verwertung von Abfällen aus privaten Haushaltungen ist deshalb zwar nach wie vor den öffentlich-rechtlichen Gebietskörperschaften übertragen (vgl. $\$ 1_{3}$ Abs. r KrW-AbfG). Für diese Bereiche ist die Daseinsvorsorge der öffentlichen Hand aus Gründen der Schaffung und Aufrechterhaltung umweltgerechter Entsorgungsstrukturen quasi durch die Hintertür, nämlich durch die Überlassungspflichten des $\$ \mathrm{I}_{3}$ Abs. I $\mathrm{KrW}-/ \mathrm{AbfG}$ wieder eingeführt worden. Dennoch ist eins unverkennbar: Der Gesetzgeber wollte die Abfallentsorgung dort, wo Abfall als Ware gehandelt werden kann, privatisieren und in private Verantwortung legen. Aus den Leitnormen für die Entsorgungsverantwortlichkeit der $\$ \$ S$ Abs. 2 und I $\mathrm{Abs}$. I $\mathrm{KrW}-/ \mathrm{AbfG}$ ergibt sich deutlich, daß der öffentlichen Hand nur noch eine Reservefunktion für die Entsorgung von Abfällen dort zukommen soll, wo anders eine effiziente und umweltverträgliche Entsorgung von Abfällen nicht möglich ist ${ }^{10}$. Ergänzt wird die weit-

7 So die Kennzeichnung bei Weidemann, in: Brandt/Ruchay/Weidemann, Kreislaufwirtschafts- und Abfailgesetz, Kommentar, Loseblatt, Stand: is.4. 1998, $\$ 4$ Rdnr. 3 .

$\delta$ Weidemann, ebd. (Fn. 7).

9 So: Petersen/Rid, Das neue Kreislaufwirtschafts- und Abfallgeserz, NJW I995, 7, 8.

I0 In diesem Sinne etwa Petersen/Rid, NJW r 995 (Fn. 9), 8; Frenz, KrW-/AbfG, 1996, Einleitung, Rdnr. 1 1 ; vgl. auch Weidemann, Einfuhrung, in: Kreislaufwirtschafts- und Abfallrecht, 1997, XXVf. 
gehende Privatisierung durch Regelungen über Abfallwirtschaftskonzepte und -bilanzen als Instrumente der Selbstkontrolle und -motivation ( $\mathbb{S}$ 1 8 , $19 \mathrm{KrW}-1$ $A b f G)^{\prime \prime}$. Über diese Instrumente sollen Betriebe veranlaßt werden, im Wege der Selbstreflexion ihre abfallrechtlichen Verwertungs- und Beseitigungspflichten zu organisieren und auf diese Weise zu einer Optimierung der Kreislaufwirtschaft als Oberziel des KrW-/AbfG bcitragen. Damit sollen nicht zuletzt Ressourcen geschont, Abfälle nach Menge und Schädlichkeit vermindert und Kosten für aufwendige Verwertungs- und Beseitigungsverfahren gespart werden.

- Der Umsetzung der genannten Prinzipien dienen die einzelnen weiteren Instrumente des Abfallrechts. Dabei ist allerdings darauf hinzuweisen, daß das $\mathrm{KrW}-$ AbfG nicht, wie es die Verschiebung der Verantwortung für die Verwertung und Beseitigung von Abfällen weg von der öffentlichen Hand hin zur privaten oder der Gedanke der Kreislaufwirtschaft und des Vermeidungs- und Verwertungsvorrangs nahelegen könnten, ausschließlich Wirtschaftsverwaltungsrecht darstellt; Abfallrecht ist nach wie vor auch Umweltschutzrecht. Das gilt insbesonderc für die Beseitigungsphase und die hierauf bezogenen Regelungen. Durch die Bestimmungen über die Abfallwirtschaftsplanung ( $\$ 29 \mathrm{KrW}-/ \mathrm{AbfG})$, den Anlagenzwang für die Bescitigung ( $\$ 27$ Abs. I KrW-/AbfG), die Regelungen über die Zulassung von Abfallanlagen und deren Verpflichtung auf die Einhaltung des Standes der Technik

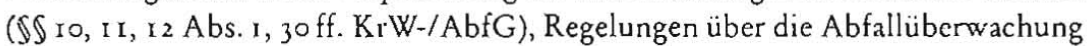
( $\$ S_{4}$ off. KrW-/AbfG) und nicht zuletzt durch Verpflichtung jedes Beseitigungsverfahrens darauf, daß die Anforderungen des Wohls der Allgemeinheit zu beachten sind ( $\$$ ro Abs. I, $4 \mathrm{KrW}-/ \mathrm{AbfG}$ ), wird die Beseitigung vor allem unter Umweltaspekten reglementiert. Markt und Marktwirtschaft und private Verantwortung spielen hier kaum, Umweltanforderungen hingegen eine hcrausragende Rolle. Das Abfallrecht kann nach alledem weder allein dem Wirtschaftsverwaltungsrecht noch allein dem Umweltschutzrecht zugeordnet werden Durch die Privatisierungsstrategie des Gesetzgebers einerseits und die Verpflichtung auf den Umweltschutz andererscits nimmt es vielmehr heute eine Zwitterstellung ein: Es handelt sich um Wirtschaftsverwaltungs- und Umweltrecht ${ }^{\prime 2}$.

Im folgenden soll überprüft werden, ob die genannten neuen Zielsetzungen des Abfallrechts wirklich rechtlich verbindlich sind und damit unmittelbar vollzogen werden können oder ob es sich insoweit nicht zumindest überwiegend um lediglich symbolhafte gesetzgeberische Zielsetzungen mit der Folge handelt, daß der Gedanke der Kreislaufwirtschafr, der eine Vcrmeidung und hochwertige sowie schadlose Verwertung von Abfällen ebenso cinschließt wie bei der Produktion von Wirtschaftsgütern das Denken vom Abfall her, lediglich als Appell zu verstehen ist und damit zwar einen Anstoß in Richtung auf eine ressourcenschonende Abfallbewirtschaftung geben und die Kreislaufwirtschaft so in Gang setzen kann, einen öffentlich-rechtlichen Vollzug in Form von verbindlichen und umsetzbaren Anordnungen an Produzenten oder Abfallerzeuger und -besitzer indessen nicht ermöglicht. Dazu soll zunächst der Frage nachgegangen werden, inwieweit die abfallrechtliche Zielhierarchie des $\S_{4}$ Abs. I KrW-/AbfG und die damit intendierte Einführung einer Kreislaufwirtschaft unmittelbar verbindliche Rechtspflichten für Produzenten und Abfallerzeuger und -besitzer auslöst (dazu 3.). Danach wird der Frage nachgegangen, welche rechtliche Qualität die Einführung der Produktverantwortung in $\$ 22 \mathrm{KrW}-/ \mathrm{AbfG}$ hat und oh sich hieraus unmittelbare Rechtspflichten für die Produzenten von Wirt-

\footnotetext{
1t Frenz, KrW-/AbfC; (Fn. ro), Finleicuag, Rdnr. 13.

12 Vgl. etwa Weidemann, KrW-/AbfG (Fn. ro), Einfuhrung, wo es heißt: „Der Abfall wird zum ,Zwitter des Wirtschaftsverwaltungsrechtsc."
} 
schaftsgütern ableiten lassen (dazu 4.). Schließlich soll auf die Zuweisung abfallrechtlicher und -wirtschaftlicher Verantwortlichkeiten an Private und ihre Unterscheidung von den Pflichten der öffentlichen Hand eingegangen werden; dabei spielt die Frage der Abgrenzung der Verantwortlichkeiten über den neuen Abfallbegriff und die Sonderung von Abfällen zur Verwertung von Abfällen zur Beseitigung eine besondere Rolle (dazu 5.). Der Beitrag schließt mit einer Zusammenfassung der Ergebnisse und einer abschließenden Bewertung (6.).

\section{Kreislaufwirtschaft als Ziel des Abfallrechts - symbolischer Akt oder ver- bindliches Recht?}

Im Zentrum des neuen Abfallrechts steht der Gedanke der Kreislaufwirtschaft. Abfälle, so soll dieser Begriff besagen, sollen nach Möglichkeit nicht beseitigt, sondern als Rohstoffe oder Rohstoffsubstitute im Wirtschaftskreislauf gehalten werden. Zur Realisierung dieses Prinzips schreibt $\${ }_{4}$ Abs. x $\mathrm{KrW}$-/AbfG eine Rangfolge von Maßnahmen in bezug auf Abfälle vor: $\rrbracket_{4}$ Abs. I KrW-/AbfG verlangt, daß Abfälle »in erster Linie « $z$ u vermeiden und »in zweiter Linie« zu verwerten sind, wobei die stoffliche und energetische Verwertung als gleichrangige Verwertungsarten genannt sind. Erst dann, wenn weder eine Vermeidung noch eine Verwertung möglich ist, soll nach dem Gedanken der Kreislaufwirtschaft cine Beseitigung von Abfällen stattfinden. Diese hat $₫ s$ Abs. $5 \mathrm{KrW}-/ A b f G$ zufolge nur dann Vorrang vor der Verwertung, wenn die Beseitigung die umweltverträglichere Lösung darstellt. Ergänzt wird diese Zielhierarchie durch die Regelungen über die Zuweisung abfallwirtschaftlicher

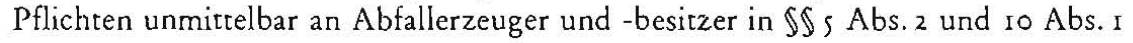
$\mathrm{KrW}-/ \mathrm{AbfG}$, die Bestimmungen über die Verwertungspflichten in $\$ s Abs. 4 und s $\mathrm{KrW}-/ \mathrm{AbfG}$ sowie die Regelungen der Produktvetantwortung in $\$ \$ 22 \mathrm{ff}$. $\mathrm{KrW}-/$ AbfG. In bezug auf alle diese Regelungen stcllt sich die Frage, ob das Leitprinzip des $\mathrm{K}_{\mathrm{rW}}$-/AbfG, nur noch »den letzten Dreck «, also das zu beseitigen, was nicht mehr für andere wirtschaftliche $Z$ wecke verwendet werden kann, hierdurch wirklich umgesetzt und damit eine effiziente Kreislaufwirtschaft initiert wird oder ob nicht vielmehr gerade die Grundphilosophie des neuen Rechts eher im Symbolischen verbleibt, weil es sich bei den einzelnen Rechtspflichten, durch die das Prinzip der Kreislaufwirtschaft rechtlich verankert werden soll, im wesentlichen um Appellpflichten oder doch um solche Pflichten handelt, die löchrig wie ein Schweizer Käse sind und damit im Konfliktfall eine Umsetzung der Prinzipien der Kreislaufwirtschaft über rechtlich verbindliche Anordnungen an Abfallerzcuger und -besitzer gerade nicht ermöglichen.

\section{I. Das Vermeidungsgebot als symbolischer Akt}

Das Vermeidungsgebot ist durch die Neuregelung in $\rrbracket_{4}$ Abs. I KrW-/AbfG zum obersten Ziel der Abfallgesetzgebung aufgestiegen. Dem Wortsinn nach bedeutet Abfallvermeidung, daß Abfall gar nicht erst anfällt. Nur Abfall, der nicht entsteht, ist vermiedener Abfall's. In diese Richtung deutet auch $\rrbracket_{4}$ Abs. I $\mathrm{Nr}$. I $\mathrm{KrW}-/ \mathrm{AbfG}$, wonach die Vermeidung insbesondere durch Verminderung der Menge und der

13 So mit Rechr: Weidemann, in: Brandt/Ruehay/Weidemann, KrW-/AbfG (Fn. 7), $\$ 4$ Rdnr. 57 . 
Schädlichkeit der Abfälle zu geschehen hat. Damit sind zwei Gesichtspunkte angesprochen, nämlich die Entstehung des Abfalls überhaupt und die Entstehung von Abfällen, die wegen ihrer Schädlichkeit besonders schwierig zu verwerten oder zu beseitigen sind. Das Vermeidungskonzept des Gesetzgebers wird dadurch - und zwar abschließend - beschrieben ${ }^{14}$. Idealziel des Gesetzes ist hiernach, daß sein Regelungsgegenstand, nämlich Abfall, wegfällt' ${ }^{15}$. Dieses Vermeidungskonzept wird in $\$ 4$ Abs. 2 $\mathrm{KrW}-/ \mathrm{AbfG}$ in Form von Beispielen näher erläutert. Maßnahmen zur Vermeidung von Abfällen sind danach insbesondere die anlageninterne Kreislaufführung von Stoffen, die abfallarme Produktgestaltung sowic ein auf den Erwerb abfall- und schadstoffarmer Produkte gerichtetes Konsumverhalten. Nach der Vorstellung des Gesetzgebers setzt die Vermeidung von Abfällen bei der Produktion an und setzt sich im Konsumverhalten fort. Damit wird einerseits an die Produktverantwortung erinnert, die darauf abzielt, durch anlageninterne Kreislaufführung sowie abfallarme Produktgestaltung zum einen die Abfallmenge und ihre Schädlichkeit bei der Produktion zu reduzieren, zum anderen aber auch dazu beizutragen, daß bestimmte Produkte, die insgesamt oder in einzelnen Bestandteilen später zu Abfällen werden, überhaupt nicht mehr in den Wirtschaftskreislauf eingebracht und Konsumenten nicht mehr angeboten werden. Das KrW-/AbfG nimmt auch den Verbraucher in den Blick. Dieser wird aufgefordert, sein Verhalten so zu verändern, daß durch seineo Konsum möglichst wenig und möglichst schadstoffarme Abfälle anfallen.

Die Zielhierarchie des $\$_{4}$ Abs. I KrW-/AbfG konstituiert freilich für sich allein noch keine unmittelbar vollziehbaren Rechtspflichten. Schon die Überschrift des $\$ 4$ $\mathrm{KrW}-/ \mathrm{AbfG}$, in der von "Grundsätzen der Kreislaufwirtschaft « die Rede ist, verdeutlicht, daß $\$ 4 \mathrm{KrW}-/ \mathrm{AbfG}$ lediglich programmatischen Charakter hat ${ }^{16}$. Unmittelbar vollziehbare Rechtspflichten enthält $\rrbracket_{4} \mathrm{KrW}-/ \mathrm{AbfG}$ nicht ${ }^{17}$. Rechtspflichten für den Bürger, Abfälle zu vermeiden, lassen sich aus $\$ 4$ Abs. I, $2 \mathrm{KrW}-/ \mathrm{AbfG}$ nicht ableiten. Die Regelung ist nicht für den Bürger, sondern lediglich für den administrativen Normgeber verbindlich ${ }^{18}$. Auch im Rahmen von Einzelfallentscheidungen kann $\$ 4$ Abs. $1 \mathrm{KrW}-/ \mathrm{AbfG}$ unmittelbar aus sich selbst heraus keinerlei Bedeutung zukommen; nur soweit die Normen, die zu einem Eingreifen ermöglichen, selbst einen Vermeidungsvorrang enthalten, ist dieser über Einzelfallanordnungen konkretisierund rechtlich umsetzbar"s.

Insgesamt läßt sich damit feststellen, daß es sich bei $\${ }_{4} \mathrm{Abs}$. I $\mathrm{KrW}-/ \mathrm{AbfG}$ und dem darin enthaltenen Vorrang der Vermeidung von Abfällen als oberstem Ziel der Kreislaufwirtschaft lediglich um eine Norm mit programmatischem Charakter handelt, die einer Konkretisierung durch andere Regclungen bedarf.

\footnotetext{
14 Kunig, in: Kunig/Paetow/Versteyl, KrW-/AbfG,I9y8), \$4 Rdnr. I3.

is Kunig, ebd. (Fn. 14).

16 In diesem Sinnc Fluck, KrW-/AbfG, Kommentar, 1996ff., $\$ 6$ Rdnr. 66; Kunig, in: Kunig/Paetorw/Versteyl, KrW-/AbfG, 1998, $\$ 4$ Rdnr. 2; Tettinger, Recheliche Baustcine eines modernen Abfallwirtschafrsrechts, DVBl 1995, 213, 217 ; ahnlich Weidemann, U1nweltschurz durch Abfallrecht - cine kritische Bewertung des neuen Krcislaufwirtschafts- und Abfallgescrzes, NVwZ 1995, 631, 633: wntauglicher Versuch $\alpha$, Formelkompromisse $\alpha$.

17 Frenz, KrW-/AbfG (Fn. 10), $\oint_{4}$ Rdnr. 4; Kunig, ebd. (Fn. 16)

18 Kunig, ebd. (Fn. 16).

19 Kunig, ebd.(Fn. 16); Petersen/Rid, NJW x995 (Fn. 9), 7, 9 f.
} 
In diesem Zusammenhang kommt vor allem $\$ s$ Abs. I KrW-/AbfG Bedeutung zu. In \$s $\mathrm{KrW}-/ \mathrm{AbfG}$ sind die Grundpflichten der Kreislaufwirtschaft zusammengefaßt. Als Grundpflicht läßt sich dabei in Anlehnung an das Immissionsschutzrecht, aus dem dieser Begriff stammt, eine Norm bezeichnen, die nicht bloß Maßstabsnorm für die Erteilung von Genehmigungen ist, sondern unmittelbare Verhaltenspflichten für den Anlagenbetreiber begründet ${ }^{20}$. Grundpflichten bedürfen zu ihrer Wirksamkeit nicht erst der Konkretisienung durch eine Rechtsverordnung oder andere untergesetzliche Vorschriften, sondern begründen unmittelbar aus sich selbst heraus Verhaltenspflichten". Bei Zugrundelegung dieses Begriffs der Grundpflicht enthält $\ 5$ Abs. r KrW-/AbfG für das Vermeidungsgebor gerade keine unmitcelbar geltende Rechtspflicht. Hierin wird nämlich klargestellt, daß konkrete Rechtspflichten zur Abfallvermeidung sich allein aus $\$ 9 \mathrm{KrW}-/ \mathrm{AbfG}$ sowie aus Rechtsverordnungen nach $\$ \$ 23,24 \mathrm{KrW}-/ \mathrm{AbfG}$ ergeben können. Die an alle Abfallerzcuger und -besitzer gerichteten Grundpflichten der Kreislaufwirtschaft sind, was das Vermeidungsgebot angeht, in $\$ ;$ Abs. I KrW-/AbfG deshalb ebenfalls nicht als konkrete, vollziehbare Rechtspflichten des Einzelnen ausgestaltet ${ }^{22}$. Die Norm verdeutlicht lediglich, durch welche Vorschriften das $\mathrm{KrW}$-/AbfG die Programmatik des $\rrbracket_{4} \mathrm{Abs}$. I Nr. I KrW-/ AbfG, also den Vermeidungsvorrang, umzusetzen erlaubt; eine Grundpflicht enthält die Regelung deshalb kcineswegs ${ }^{23}$. Vielmehr handelt es sich allenfalls um eine Norm mit Appellcharakter ${ }^{24}$.

Betrachtet man allein die Regelungen über die Grundsätze und Grundpflichten der Kreislaufwirtschaft, läßt sich damit insgesamt feststellen, daß hierin das Vermeidungsgebot als oberstes Ziel der Kreislaufwirtschaft keine rechtlich verbindliche Ausgestaltung erfahren hat. Unmittelbare Rechtspflichten für Abfallerzeuger und besitzer folgen hieraus nicht. Es verbleibt bei einem appellhaften Charakter. Damit aber sind diese Normen typische symbolische Gesetzgebungsakte.

\subsection{Konkretisierung des Vermeidungsgebots in $\int 9 \mathrm{KrW}-/ A b f G$ oder durch Rechtsverordnungen nach $\iint_{23}, 24 \mathrm{KrW}-/ A b f G$ ?}

Dieser Befund würde sich freilich dann relativieren, wenn sich aus $\$ 9 \mathrm{KrW}-/ \mathrm{AbfG}$ unmittelbar oder aus Rechtsverordnungen nach $\$ \$ 23,24 \mathrm{KrW}-/ \mathrm{AbfG}$ Rechtspflichten zur Vermeidung von Abfällen ableiten ließen. Auch in diesen Vorschriften ist allerdings die Rechtspflicht zur Vermeidung von Abfällen nicht verankert; unmittelbar für Erzeuger und Besitzer von Abfällen oder Konsumenten geltende Rechtspflichten lassen sich hieraus mit dem Ziel eines Vermeidungsvorrangs kaum ableiten:

$20 \mathrm{Vgl}$. Kutscheidt, in: Landmann/Rohmer, Umweitrecht I, $\$ s$ BImSchG, Rdnr. 8 f.; Jarass, Bundesimmissionsschurzgesetz, 3. Aufl.1995, \&S Rdnr. I.

21 Hoffmann, Abfallrechtliche Produktverantwortung, DVB]. 1996, 898, 899; Weidenann, in: Brandt/ Ruchay/Weisemann, KrW-/AbfG (Fn. 7), \&s Rdnr. 22 f

22 Beckmann, Produktverantwortung, UPR 1996, 4 r, 45; Fluck, KrW-/AbfG (Fn. I6), \$s Rdnr. 7r; Frenz, KrW-/AbfG (Fn. 10), \$s Rdnr. 4; Kunig, In: Kunig/Paetow/Versteyl, KrW-/AbfG (Fn. 14); \$s Rdnr. 7; Schink, Von den Schwierigkeiten der Kommunen mit dem Kreislaufwirtschafts- und Abfallgesecz, ZG 1996, 97, 106; Verstcyl/Wendenburg, Das Kreislaufwirtschafts- und Abfallgesetz, NVwZ 1994, 837; Weidemann, ia: BrandL/Ruchay/Weidemann, KrW-/AbfG (En. 7), \$s Rdnr. 26; ders., Anforderungen an die Kreislaufwirtschaft, in: Hoppe/Bauer/Faber/Schink (Hrsg.). Auswirkungen des Kreislaufwirtsehafts- und Abfallgeseczes auf die öffentlich-rechtlichen Entsorgungsträger, 1996, S. 49, s2.

23 Kunig, ebd. (Fn. 22).

24 Schink, ZG 1996 (Fn. 22), 106; Versreyi/Nendenburg, NVwZ 1994 (Fn. 22), 837 
$\$ 9 \mathrm{KrW}-/ \mathrm{AbfG}$ betrifft die Pflichten der Betreiber von nach Immissionsschutzrecht genehmigungsbedürftigen und nicht genehmigungsbedürftigen Anlagen. Nach $\$ 9$ Satz I KrW-/AbfG richten sich die Vermeidungs-, Verwertungs- oder Beseitigungspflichten für die Betreiber solcher Anlagen nach den Vorschriften des BImSchG. Für genehnigungsbedürftige Anlagen gelten damit für die Pflicht zur Abfallvermeidung die Regelungen des $\$ s$ Abs. 1 Nr. 3 BImSchG unmittelbar; für nichr genehmigungsbedürftige Anlagen bedarf es hingegen gemäß $\$ 22$ Abs. I Satz 2 BImSchG einer Verordnung, damit die Anforderungen des $₫ 5$ Abs. 1 Nr. 3 BImSchG entsprechend gelten. Nach $\$ 9$ Satz I KrW-/AbfG werden damit die sich aus dem KrW-/AbfG für die Abfallvermeidung, Verwertung und Beseitigung für die Betreiber von nach Immissionsschutzrecht zu behandelnden Anlagen ergebenden Rechtspflichten durch die Regelungen des BImSchG verdrängt ${ }^{2}$. Das gilt indessen lediglich für die anlagenbezogenen Anforderungen; die stoffbezogenen ergeben sich weiter aus dem $\mathrm{KrW}$-I AbfG ( $\$ 9$ Satz 2 KrW-/AbfG) und sind in bezug auf die anlageninterne Verwertung durch Rechtsverordnung nach $\$ 6 \mathrm{Abs} .1$ und $7 \mathrm{KrW}-/ \mathrm{AbfG}$ festzulegen ( $\$ 9$ Satz 3 $\mathrm{KrW}-/ \mathrm{AbfG})^{26}$. Dabei sind stoffbezogen im Gegensatz zu anlagenbezogen solche Anforderungen, die Regelungen über den Umgang mit einem Stoff aufstellen, unabhängig davon, ob er in einer Anlage verwendet oder sonstwie genutzt wird ${ }^{27}$.

Fragt man, ob die anlagenbezogenen Regelungen des $\ s$ Abs. I Nr. 3 BImSchG einen Vorrang der Vermeidung für die Betreiber von nach Immissionsschutzrecht genehmigungsbedürftigen Anlagen enthalten, so läßt sich feststellen, daß dies nicht der Fall ist: Nach $\$ 5$ Abs. I Nr. 3 BImSchG sind Abfälle zu vermeiden, es sei denn, sie werden ordnungsgemäß und schadlos verwertet oder, soweit Vermeidung und Verwertung technisch nicht möglich oder unzumutbar sind, als Abfälle ohne Beeinträchtigung des Wohls der Allgemeinheit beseitigt. Schon vom Wortlaut der Norm her gibt es damit keinen Vermeidungsvorrang als Pflicht des Anlagenbetreibers. Der Anlagenbetreiber hat vielmehr die Wahl zwischen der Vermeidung und der Verwertung ${ }^{28}$. Eine unmittelbar geltende Rechtspflicht, Abfälle vorrangig zu vermeiden, folgt damit aus $\$$ S Abs. I Nr. 3 BImSchG keineswegs. Darüber hinaus entfällt die Pflicht zur Verıneidung dann, wenn sie technisch nicht möglich ist oder wenn die Vermeidung unzumutbar ist. Eine Vermeidungspflicht gibt es damit etwa dann nicht, wenn die Belastung des konkreten Anlagenbetreibers durch die Vermeidung unangemessen im Verhältnis zum Nutzen ist, der sich aus der Vermeidung ergibt. Dabei müssen im einzelnen die Nachteile der Vermeidung für den Anlagenbetreiber gegenüber der Abfallbeseitigung mit den Vorteilen für die Umwelt abgewogen werden. Soweit die Kosten der Verwertung die der Beseitigung erheblich übersteigen, wird in der Regel die Vermeidung unzumutbar sein; dasselbe gilt, wenn Vermeidungskosten zu einem nicht mehr marktfähigen Preis des Produktes führen ${ }^{29}$.

Bei näherem Hinsehen zeigt sich damit, daß es einen Vermeidungsvorrang für die Betreiber von nach Immissionsschutzrecht genehmigungsbedürftigen Anlagen nicht gibt. Der Regelungsmechanismus des $\mathrm{KrW}$-/AbfG für diesen Bereich führt dazu, daß

25 Frenz, KrW-/AbfG (Fn. 10), \$9 Rdnr. 1; Pactow, in: Kunig/Paetow/Versteyl, KrW /AbfG (Fn. I4), \$9 Rdnr. 3,6 .

$26 \mathrm{Vgl}$. nur Paetow, in: Kunig/Paetow/Versteyl, KrW-/AbfG (Fn. 14), \$9 Rdnr. 7.

27 Vgl. Fluck, KrW-/AbfG (Fn, r6), \$9 Rdnr. Is6.

28 Kutscheidr, Die Neuregelung der Abfallvermeidungs- und -beseitigungspflichten bei industriellen Betrieben, NVwZ 1986, 623; Jarass, BImSch G (Fn. 20), \&; Rdnr. 76; ders., Die jüngsten Anderungen des Immissionsschutzrechts, NVwZ, 1986, 608; Sellner, Immissionswchutzrecht und Industrieanlagen, 2 . Aufl. 1988, Rdnr. 64. A. A. Rebentisch, Technische Konerolle in staarlicher Verantwortung, Lil'R Iy $87,4{ }^{C} 3$. 29 Zum Vorstehenden Jarass, BImSchG (Fn. 20), $\$$ s Rdnr. 75 . 
der vollmundig in $\S_{4}$ Abs. I Nr. r KrW-/AbfG kreierte Grundsatz des Vermeidungsvorrangs im Konkreten, nämlich dort, wo im Rahmen der Produktion von Stoffen eine Vermeidung technisch möglich ist, vor der Verwertung gerade keinen Vorrang hat und auch in bezug auf die Beseitigung nur dann gefordert wird, wenn Vermeidung oder Verwertung wirtschaftlich zumutbar sind. Daß dies eine echte Rechtspflicht für Anlagenbetreiber darstells, bei der Produktion Abfälle zu vermeiden, wird man wohl kaum sagen können. Für einen der wichtigste Bereiche, in dem eine Vermeidung von Abfällen wirklich Erfolge bringen kann, verbleibt es damit bei einer eher symbolhaften Regelung in $\S_{4} \mathrm{Abs}$. I KrW-/AbfG, die nicht in echte Rechtspflichten umgesetzt ist.

Hervorzuheben ist dabei freilich, daß von dieser Symbolik durchaus positive Wirkungen ausgehen können. Der Gesetzgeber hat im KrW-/AbfG verdeutlicht, daß Vermeidung von Abfällen für ihn bei der Produktion oberste Priorität besitzt. Damit fordert er alle Betriebe des produzierenden Gewerbes auf, dieses Ziel auch wirklich ernst zu nehmen und in der Produktion umzusetzen. Ob davon allein allerdings große Wirkungen ausgehen können, erscheint mehr als zweifelhaft. In der Praxis der Unternehmen dürfte es wohl weniger der gesetzgeberische Appell sein, Abfälle zu vermeiden, der solche Überlegungen auslöst. In erster Linie entscheiden vielmehr Kostenüberlegungen. Ist es kostengünstigcr, in eine Anlagc mit dem Ziel zu investieren, Abfälle zu vermeiden, statt sie zu verwerten oder zu beseitigen, wird dieser Weg beschritten. Die Vermeidungsphilosophie des Gesetzgebers dürfte dabei kaum eine Rolle spielen.

\subsubsection{Konkretisierung des Vermeidungsgebotes durch Rechtsverordnungen nach $\iint 23,24 \mathrm{KrW}-/ \mathrm{AbfG}$}

Der vorstehende Befund hat ergeben, daß sich die Umsetzung des Vermeidungsziels im Zweifelsfall nur durch den Erlaß von Rechtsverordnungen auf der Grundlage der $\mathbb{S} 22 \mathrm{ff}$. KrW-/AbfG erreichen läßt ${ }^{30}$. Durch Verordnungen aufgrund der $\mathbb{\$} 23,24$ $\mathrm{K}$ W-/AbfG können konkrete Verantwortlichkeiten für bestimmte Produkte festgelegt werden. Hervorzuheben ist dabei, daß in solchen Verordnungen Vcrwendungsund Beschaffenheitsgebote $(\$ 23 \mathrm{Nr} .1,3)$, Beschaffenheitsverbote $(\$ 23 \mathrm{Nr}$. 2), Kennzeichnungspflichten $\left(\$_{23} \mathrm{Nr} .4-7\right)$, Rückgabe- und Rücknahmepflichten $(\$ 24$ Abs. I Nr. I-3), Nachweispflichten ( $\$ 24$ Abs. I Nr. 4), Bestimmungen über die Kostenlast $(\$ 24$ Abs. 2 Nr. I) und organisatorische Fragen der Entsorgung ( $\$ 24$ Abs. 2 Nr. $2-4$ ) geregelt werden können. Verwendungs- und Beschaffenheitsgebote, Beschaffenheitsverbote und Kennzeichnungspflichten sind dabei als Mittel der Umsetzung des Ziels der Abfallvermeidung prinzipiell geeignet. Über Verwendungs- und Beschaffenheitsgebote bzw. Beschaffenheitsverbote kann Einfluß auf die Produktgestaltung genommen werden: Müssen Produkte in einer bestimmten Weise, etwa als Mehrwegsysteme ausgestaltet und verwendet werden, hat dies Einfluß auf die Abfallmenge, die zu verwerten oder zu beseitigen ist. Beschaffenheitsgebote und Beschaffenheitsverbote nehmen Einfluß auf die Menge des Materials und die Art des Materials, aus dem Produkte hergestellt werden Sie können damit zum cinen zu einer Verringerung der zu verwertenden oder zu beseitigenden Abfallmengen, zum anderen aber auch des Schadstoffgehaltes der Abfälle beitragen und sind insofern vermeidungsrelevant.

30 In diesem Sinne etwa Bleicher, Das neue Krcislaufwirtschafts- und Abfallgcset\%, Landkreis 1994, 552, 553; Queitsch, Kreislaufwirtschafts- und Abfallrccht, 1995, Anm. 2 zu $\S_{4} \mathrm{KrW}-/ \mathrm{AbfG}$ (S. Ic6). 
Bestimmungen über Kennzeichnungspflichten richten sich in erster Linie an den Verbraucher, der auf diese Weise zu einem "abfallärmeren" Konsumverhalten veranlaßt werden kann. Schließlich ist von besonderer Bedeutung die Bestimmung über die Kostenlast. Müssen Erzeuger oder diejenigen, die bestimmte Produkte in den Verkehr bringen, die Kosten für die Verwertung oder Beseitigung im Rahmen ihrer Produktverantwortung tragen, hat dies zur Folge, daß marktwirtschaftliche Anreize dafür geschaffen werden, bestimmte Produkte erst gar nicht in den Verkehr zu bringen bzw. so in den Verkehr zu bringen, daß die Kosten für die Verwertung oder Beseitigung der Abfälle, dic durch das Produkt letztlich verursacht werden, minimiert werden. Dieser marktwirtschaftliche Anreiz kann deshalb einen Vermeidungserfolg auslösen, und zwar auch ohne daß das Inverkehrbringen bestimmter Produkte oder Produktarten oder Materialien ausdrücklich untersagt wird.

Bei näherem Hinsehen ist in der bisherigen Verordnungspraxis des Bundes lediglich der letzte Aspekt, nämlich der der Kostenpflicht der Erzeuger und derjenigen, die Produkte in den Verkehr bringen, für die Vermeidungsverpflichtung fruchtbar gemacht worden. Eine unmittelbare Rechtspflicht, bestimmte Abfälle zu vermeiden, ist hingegen nur in Ansätzen verwirklicht worden:

Der Prototyp einer Verordnung nach $\mathbb{S}\{23,24 \mathrm{KrW}-/ \mathrm{AbfG}$ ist die Verpackungsverordnung ${ }^{31}$. Das Vermeidungsgebot war in der VerpackV bereits in ihrer ursprünglichen Fassung von 1991 verankert. Das Ziel der Vermeidung von Verpackungen war scinerzeit in $\$ 1$ Abs. 2 VerpackV angesprochen. Dort hieß es, daß Abfälle aus Verpakkungen dadurch zu vermeiden ist, daß Verpackungen

- nach Volumen und Gewicht auf das zum Schutz des Füllgutes und auf das zur Vermarktung unmittelbar notwendige Maß beschränkt werden,

- so beschaffen sein müssen, daß sie wieder befüllt werden können, soweit dies technisch möglich und zumutbar sowie vereinbar mit den auf das Füllgut bezogenen Vorschriften ist und

- stofflich verwertet werden, soweit die Voraussetzungen für eine Wiederbefüllung nicht vorliegen.

Einen Vermeidungsvorrang kannte die VerpackV in $\$$ I nicht. Dieser ist erst durch $\$$ I Satz 2 VerpackV 1998 rechtlich festgeschrieben worden. Allerdings ist es auch bei der VerpackV in der Neufassung vom August 19998 bei dieser - eher programmatischen Formulierung geblieben. Rechtliche Regelungen, die mit unmittelbar verbindlicher Wirkung geeignete Maßnahmen zur Erreichung des Ziels der Abfallvermeidung festschreiben würden, enthalten hingegen weder die VerpackV aus dem Jahre 1991 noch die VerpackV in der Änderungsfassung von August 1998. Notwendig hierfür wäre, daß konkrete Vermeidungspflichten etwa in bezug auf Umverpackungen oder überflüssige Verkaufsverpackungen festgeschrieben würden und daß für die Hcrstellung der Verpackungen konkrete Vorschriften über ihre Zusammensetzung gemacht würden. Nur auf diese Weise könnten Abfälle, dic aus Verpackungsmaterialien entstehen, in ihrer Menge und ihrem Schadstoffgehalt wirklich reduziert werden. Darüber hinaus wärc es dringend geboten, vom Rücknahmesystem DSD, das nach $\$ 6$ Abs. 3 VerpackV die Rücknahmepflicht des Handels für Verkaufsverpackungen entfallen läßt, nähere Darlegungen zum Verbleib der Verpackungen zu verlangen; schließlich müßten von den Garantiegebern Verwendungsnachweise verlangt sowie grundsätzliche Kontrollrechte der Behörden eingeräumt werden ${ }^{32}$. Solche Forderungen enthielt die ursprüngliche Fassung der VerpackV nicht. Insbesondere fehlten

31 Verordnung über die Vernseidung und Verwertung von Verpackungsabfällen (Verpackungsverordnung VerpackV) von 1. 2. Juni 1991 (BGBl. I, (234), Neufassung vom 21.8. 1998, BGBl. I, 2379.

32 Zu dicsen Forderungen vgl. etwa Berg/Hósch, Die Produktverantwortung nach $\$ 22 \mathrm{KrW}-/ \mathrm{AbfG}$, Jb. UTR $1997,83,103$. 
Regelungen über die Zusammensetzung von Verpackungsmaterialien. Eine solche

Regelung ist erst durch $\mathbb{I}_{12}$, I $_{3}$ VerpackV ${ }_{199} 8$ eingeführt worden. Die Regelungen über die Mehrwegquote sind unzureichend. So gibt es zwar eine Pfanderhebungspflicht für Getränkeverpackungen und Verpackungen von Wasch- und Reinigungsmitteln sowie Dispersionsfarben, soweit diese keine Mehrwegverpackungen sind ( $\$ 8$ VerpackV). Diese Regelung findet indessen keine Anwendung auf die Hersteller oder Vertreiber der Verpackungen, die sich an einem Rücknahmesystem nach $\$ 6$ Abs. 2 VerpackV beteiligen ( $\$ 8$ Abs. I VerpackV). Die Pfanderhebungsregelung ist damit in der Bundesrepublik nahezu wirkungslos. Denn alle diejenigen, die Einwegverpakkungen für die genannten Waren in den Verkehr bringen, haben sich in der Vergangenheit am Dualen System beteiligt mit der Folge, daß ein Pfand für eine Einwegverpakkung bislang nicht erhoben worden ist. Dies indessen führt mittelbar dazu, daß besondere Anreize, Mehrwegsysteme einzuführen, über die VerpackV gerade nicht geschaffen werden, sieht man einmal davon ab, daß nach $\$ 9$ Abs. 2 VerpackV bestimmte Mehrwegquoten crreicht werden müssen. Besondere Anreize für eine Vermeidung von Verpackungen wegen des Vorrangs von Mehrwegverpackungen sind indessen nicht festzustellen. Insgesamt läßt sich konstatieren, daß die VerpackV, was den Vermeidungsgedanken angeht, über unmittelbare Rechtspflichten kaum wirksam geworden ist. Wirkungen gingen bislang lediglich von der Regelung über die Kostenverantwortlichkeit der Betreiber von Rücknahmesystemen sowie derjenigen aus, die Umverpackungen und Transportverpackungen in den Verkehr bringen. Diese Regelungen haben dazu geführt, daß insbesondere der Anteil der Umverpakkungen sowie der Transportverpackungen stark zurückgegangen ist. Ursache dafür ist letztlich, daß für diese Verpackungsarten ein Rücknahmesystem nach dem Muster des Dualen Systems nicht etabliert worden ist. Die Kosten müssen damit unmittelbar von den Herstellern und Vertreibern getragen werden. Dies war Veranlassung dafür, auf Umverpackungen weitgehend zu verzichten und bei Transportverpackungen nach Möglichkeit wiederverwendbare Systeme einzusetzen. Im Bereich des großen Blocks der Verkaufsverpackungen ist der Kostenmechanismus und damit der marktwirtschaftliche Aspekt der VerpackV freilich nicht in gleichem Umfang wirksam geworden. Wirksam kann dieser Mechanismus allein über die Lizenzentgeltregelung des Dualen Systems werden. Hierfür enthielt die ursprüngliche VerpackV keine Vorgaben mit der Folge, daß ursprünglich allein Größe und Gewicht der Verpakkungen über die Höhe des Lizenentgelts entschieden. Dieses System ist inzwischen umgestellt worden mit der Folge, daß leichtere und leichter wiederverwendbare Verpackungen eingesetzt werden. Dies mag man als Vermeidungserfolg des marktwirtschaftlichen Systems VerpackV charakterisieren, da immerhin Menge und Schadstoffgehalt der Verpackungsabfälle abgenommen haben. Eine wirkliche Vermeidungsstrategie stellt die VerpackV damit allerdings noch nicht dar. Nach wie vor werden in großer Menge Verpackungen in den Verkehr gebracht, die an sich verzichtbar sind. Vermeidungserfolge im Bereich der Verkaufsverpackungen sind durch die VerpackV damit zwar initiiert worden; in ihrer Effizienz bleiben sie jedoch hinter Erfolgen, die durch unmittelbar verbindliche Regelungen zu erzielen wären, deutlich zurück.

Ähnliche Feststellungen wie zur Verpackungsverordnung lassen sich auch für die anderen bislang auf $\$ \$ 23$ und $94 \mathrm{KrW}$-/AbfG gestützten Verordnungen treffen:

Die Altautoverordnung ${ }^{3 j}$ erwähnt das Ziel der Abfallvermeidung nicht, sondern zielt

33 Verordaung uber die Uberlassung und umweltvertragliche Entsorgung von Altautos (Altauto-Verordnung AltautoVO) vom 4.7.1997, verkündet als Artikel r der Verordnung über die Entsorgung von Altautos und die Anpassung straßenverkehrsrechtlicher Vorschriften vom 4.7. 1997, BGBl. I, I666. 
ausschließlich darauf ab, eine umweltgerechte und ordnungsgemäße Verwertung und Beseitigung von Altautos zu regeln; sie dient dabei letzilich der Umsetzung der freiwilligen Selbstverpflichtung der Automobilindustrie zur umweltgerechten Altautoverwertung ${ }^{34}$, durch die sich die Automobilindustrie zur Rücknahme und Verwertung von Altautos verpflichtet hat. Demzufolge trifft sie Regelungen ausschließlich für die Verwertung von Altautos und setzt technische Anforderungen für Verwerterbetriebe. Eine Vermeidung von Abfällen aus Automobilen kann auch über diese Verordnung nur mittelbar, nämlich dadurch bewirkt werden, daß Automobile so konstruiert werden, daß möglichst wenig Abfälle mit einem möglichst geringen Schadstoffgehalt im Stadium des Recyclings von Altautos anfallen.

Für die Batterieverordnung"ss gilt ähnliches. Diese Verordnung, die der Umsetzung der Batterierichtlinie der EG dient ${ }^{36}$, nennt als abfallwirtschaftliches Ziel $z$ war die Abfallvermeidung, indem dort formuliert ist, daß es darum geht, den Eintrag von Schadstoffen in Abfällen durch Batterien zu verringern. In Wahrheit geht es ungeachtet dessen, daß in $\$ 1_{3}$ BattV Verbote für das In-Verkehr-bringen bestimmter Batterien enthalten sind, bei der BattV letztlich um den Aufbau eines Rücknahmeund Verwertungssystems für gebrauchte Batterien. Yin Vermeidungserfolg hinsichtlich der Menge wird überhaupt nicht angestrcbt; hinsichtlich des Schadstoffgehalts geht die BattV über das bisher aufgrund freiwilliger Vereinbarungen Erreichte ebenfalls nicht hinaus. Letztlich wird bei der BattV ein weitergehender Vermeidungserfolg hinsichtlich der Schadstoffbelastung durch Batterien deshalb voraussichtlich ebenfalls nur über marktwirtschaftliche Anreize, nämlich die Verwertungskosten für Battericn erzielt werden können.

Resümiert man, so läßt sich feststellen, daß auch die aufgrund der $\$ \$ 23,24 \mathrm{KrW}-/$ AbfG erlassenen Verordnungen das Ziel der Vermeidung allenfalls verbal zum Ausdruck bringen, daß sie jedoch besonders weitreichende Instrumente zur Verringerung der Menge oder des Schadstoffgehalts von Abfällen nicht enthalten. Ordnungsrecht setzen sie insoweit lediglich für das In-Verkehr-bringen bestimmter schadstoffbelasteter Produkte. Im übrigen setzen sie auf marktwirtschaftliche Instrumente. Besonders weitreichende Regelungen zur Abfallvermeidung und damit zur Umsetzung des obersten Ziels der Kreislaufwirtschaft sind damit gerade nicht getroffen worden. Dieses Ziel verbleibt auch über die Umsetzung durch Verordnungen nach $\$ \$ 23,24$ $\mathrm{KrW}-/ \mathrm{AbfG}$ vor allem im Symbolischen. Eine Lenkung in Richtung auf eine Vermeidung durch ordnungsrechtliche Instrumente findet nicht statt.

\subsubsection{Abfallberatungspflicht nach $\$ 38 \mathrm{KrW}-/ A b f G$ als Instrument zur Abfallvermeidung}

Fragt man danach, wie das Konsumverhalten der privaten Haushalte oder die Produktion in Gewerbebetrieben in Richtung auf die Vermeidung von Abfällen gelenkt werden kann, bleibe Ictztlich nur die Abfallberatungspflicht des $\$ 38$ Abs. I KrW-/

34 Freiwillige Selbstverpflichtung zur umweltgerechten Altautoverwertung (PKW) im Rahmen des Kreislaufwirtschaftsgesetzes vom 21. Februar 1996 in der Fassung vom November 1996, abgedruckt bci Brandt/Ruchay/Weidemann, KrW-/AbfG (Fn. 7), A i 32.I.

35 Verordnung uber die Rucknahme und Entsorgung gebrauchter Battericn und Akkumulatoren (Batterieverordnung - BatrV) vom 27.3.1998, BGB1. 1, 1658 .

36 Richtlinie 9x/ is 7/EWG des Rates uber gefahrliche Stoffe cnchaltende Batterien und Akkumulatoren vom เS. März 199 r (ABl. EG 1991, L 78, S. 38), geàndert durch RL 93/86/EWG vom 4. 10. 1993 (AB!. EG 1993, L 264 , S. s1). Dazu: Schink, in: Rengeling (Hrsg.), Handbuch des curopaischen und deutschen Umweltreches, Band 2 (HEDOR II), 1998, \$74, Rdnrn. 97 ff. 
AbfG. Nach dieser Regelung sind die Entsorgungsträger im Sinne der $\$ \$$ is, 17 und I 8 KrW-/AbfG sowie die Selbstverwaltungskörperschaften der Wirtschaft (d.h. die Industrie- und Handelskammern, Handwerkskammern und landwirtschaftlichen berufsständischen Organisationen) im Rahnien der ihnen übertragenen Aufgaben zur Information und Beratung u.a. über die Möglichkeiten der Abfallvermeidung verpflichtet; sie nehmen diese Aufgabe in Selbstverwaltung wahr. Damit wird dem Gedanken Rechnung getragen, daß sich die Ziele der Abfallvermeidung und Abfallverwertung ohne eine Beratung der Abfallproduzenten und -besitzer sowie eine hierauf zugeschnittene Öffentlichkeitsarbeit kaum verwirklichen lassen ${ }^{37}$. Der Gesetzgeber bedient sich damit "weicher "Mittel, um auf das Verhalten mit dem Ziel einer Vermcidung von Abfällen Einfluß zu nehmen. Ob die Effekte dabei allerdings besonders groß sein können, ist vor allem für den gewerblichen Bereich zu bezweifeln. Die Aufgabe der Beratung von gewerblichen Unternehmen mit dem Ziel, Abfälle zu vermeiden, obliegt den Entsorgungsträgern nach $\mathbb{S} 15,17$ und 18 $\mathrm{KrW}-/ \mathrm{AbfG}$. In erster Linie angesprochen sind damit die öffentlich-rechtlichen Entsorgungsträger, denn Verpflichtete nach $\mathbb{S} \mathrm{I} 7$ und $18 \mathrm{KrW}-/ \mathrm{AbfG}$ gibt es in der Bundesrepublik bislang nicht. Abfallwirtschaftliche Beratung, die das Ziel verfolgt, bei der Produktion das Entstehen von Abfällen zu vermeiden, ist aber in erster Linie betriebswirtschaftliche und technische Beratung. Sie setzt die Kenntris von Arbeits- und Produktionsabläufen voraus. Solche Kenntnisse besitzen die öffentlichrechtlichen Entsorgungsträger in der Regel nicht, so daß deren Beratung der gewerblichen Unternehmen kaum wirksam werden $\mathrm{kann}^{3}{ }^{3}$. Ob die Industrie- und Handelskammern dazu in der Lage sind, eine spezialisierte Beratung für jeden Industriebetrieb vorzuhalten, erscheint ebenfalls zweifel haft mit der Folgc, daß mit dem Mittel der Abfallberatung nach der Konzeption des Gesetzgebers allenfalls in Teilbereichen Erfolge für das Ziel der Abfallvermeidung erreicht werden können. Auch dies ist ein stumpfes Schwert.

\subsection{Abfallvermeidung als bloß umweltpolitische Zielsetzung}

Resümiert man, läßt sich feststellen, daß das Ziel der Abfallvermeidung ganz überwiegend ein bloßes politisches Leitprinzip ist. Verpflichtende Vorgaben dazu ergeben sich weder aus $\$ \mathrm{~S}_{4}$ und $5 \mathrm{KrW}-/ \mathrm{AbfG}$ noch - sieht man einmal von beschränkten Verboten des In-Verkehr-bringens bestimmter Produkte ab - aus den bislang zur Konkretisierung der Produktverantwortung aufgrund $\operatorname{der} \$ \$ 23,24 \mathrm{KrW}-/ \mathrm{AbfG}$ erlassenen Verordnungen. Der Gesetz- und Verordnungsgeber setzt nahczu ausschließlich auf markrwirtschaftliche Instrumente sowie die Überzeugung von Produzenten und Abfallbesitzern durch die Abfallberatung. Das Ziel der Vermeidung ist damit zwar nicht wirkungslos. Die abfallrechtliche Vorgabe der $\mathbb{\$} 4$ und $5 \mathrm{KrW}-/$ AbfG, die das Postulat der Vermeidung als oberstes Ziel der Kreislaufwirtschaft formulieren und dieses Ziel in den Grundpflichtenkatalog des $\$ 5 \mathrm{KrW}-/ \mathrm{AbfG}$ einreihen, wird damit nicht eingelöst. Der Leitgedanke der Abfallvermeidung ist ganz überwiegend nur symbolisch gemeint. Ein rechtliches Instrumentarium zu seiner Umsetzung fehlt weitestgehend. Zur Ehrenretcung des Gesetzgebers sei allerdings

37 Vgl. dazu Schwade, in: Schink/Schmektn/Schwade, Abfallgesctz des Landes NW, 2. Auflage 1996, $\$ 3$ Anm. 4.2 (S. 103); Frenz, KrW-/AbfG (Fn. 10), \$38 Fn. I; Paetow, in: Kunig/Paetow/Versteyl, KrW-1 AbfG (Fn. 14), $\$ 38$ Rdnr. 4 .

38 In diesem Sinne erwa Paetow, in: Kunig/Paetow/Versteyl, KrWW-/AbfG (Fn. I 4), \$38 Rdnr. 7, Schwade, in: Schink/Schmeken/Schwade, LAbfG NW (Fn. 37), \$3 Anm 4.2 (S. Ios ff.). 
auf folgendes hingewiesen: Ein Einwirken auf Produkrionsprozesse mit dem Ziel einer Verminderung von Abfallmengen oder des Schadstoffgehaltes von Abfällen über Verbote oder Gebote ist auch für den Gesetzgeber sehr schwierig. Über Verordnungen nach $\$ \$ 23$ und $24 \mathrm{KrW}-/ \mathrm{AbfG}$ werden sich insoweit Erfolge kaum erzielen lassen. Ob dies allerdings für den Gesetzgeber Anlaß dazu sein sollte, die Vermeidung von Abfällen zwar als Ziel zu formulieren, es jedoch weitestgehend bei einem Appellcharakter dieses Ziels zu belassen, erscheint mehr als zweifelhaft. Nicht der Appell, sondern wirtschaftliche Überlegungen sind es jedenfalls bei gewerblichen Unternehmen, die Überlegungen zur Vermeidung von Abfällen auslösen.

\section{Produktverantwortung als bloße Symbolik}

Wie erwähnt, ist die Produktverantwortung des Unternehmers eine der zentralen Neuregelungen des KrW-/AbfG. Diese in $\$ 22 \mathrm{KrW}$-/AbfG festgelegte Verantwortung des Produzenten ist das Rückgrat der privaten Verantwortung für die Abfallvermeidung und -entsorgung ${ }^{39}$; sie bildet die Grundlage dafür, daß die Entsorgungsverantwortung von Privaten, die zur Verwertung und Beseitigung von Abfällen nicht in der Lage oder willens sind, auf dazu fähige Private verlagert wird. Sie ist tragende Säule des Konzepts des Gesetzgebers, Abfallaspekte bereits bei der Produktion zu bedenken und so am Ursprung der von Abfällen ausgehenden Gefahren anzusetzen. Die Hersteller und Vertreiber von Produkten sollen über dieses Instrument im Sinne des Verursacherprinzips in die Pflicht genommen werden, da sie es sind, die über ihre Produkte die Gefahren, die bei der Abfallentsorgung bestehen, erst schaffen, während der Verbraucher solche Gefahren lediglich aktualisier $t^{\circ}$. Das Konzept des Gesetzgebers, Abfälle zu vermeiden und Ressourcen zu schonen sowie Gefahrcnpotentiale, die bei der Abfallentsorgung entstehen können, nach Möglichkeit zu minimieren, bedarf der Produktverantwortung als Grundlage.

In $\$ 22$ Abs. I Satz I KrW-/AbfG ist Produktverantwortung dahin gekennzeichnet, da $\beta$ derjenige, der Erzeugnisse entwickelt, herstellt, be- und verarbeiter oder vertreibt, zur Erfuillung der Ziele der Kreislaufwirtschaft die Produktverantwortung trägt. Zur Erfüllung dieser Produktverantwortung, so $\$ 22 \mathrm{Abs}$. I Satz $2 \mathrm{KrW}$-/AbfG, sind Erzcugnisse möglichst so zu gestalten, daß bei deren Herstellung und Gebrauch das Entstehen von Abfällen vermindert wird und die umweltverträgliche Verwertung und Beseitigung der nach deren Gebrauch entstandenen Abfälle sichergestellt ist. Näher erläutert werden die Elemente der Produktverantwortung in $\$ 22$ Abs. 2 $\mathrm{KrW}-/ \mathrm{AbfG}$. Danach umfaßt die Produktverantwortung insbesondere

- die Entwicklung, Herstellung und das In-Verkehr-bringen von Erzeugnissen, die mehrfach verwendbar, technisch langlebig und nach Gebrauch zur ordnungsgemäßen und schadlosen Verwertung und umweltverträglichen Beseitigung geeignet sind,

- den vorrangigen Einsatz von verwertbaren Abfällen oder sekundären Rohstoffen bei der Erstellung von Erzeugnissen,

- die Kennzeichnung von schadstoffhaltigen Erzeugnissen, um die umweltverträgliche Verwertung oder Beseitigung der nach Gebrauch verbleibenden Abfälle sicherzustellen, 
- den Hinweis auf Rückgabe, Wiederverwertungs- und Verwertungsmöglichkeiten oder -pflichten und Pfandregelungen durch Kennzeichnung der Erzeugnisse und

- die Rücknahme der Erzcugnisse und der nach Gebrauch der Erzeugnisse verbleibenden Abfälle sowie deren nachfolgende Verwertung oder Beseitigung ${ }^{41}$.

Nach $\ 22$ Abs. 4 KrW-/AbfG bestimmt die Bundesregierung durch Rechtsverordnungen aufgrund der $\mathbb{S} 23$ und $24 \mathrm{KrW}-/ \mathrm{AbfG}$, welche Verpflichteten die Produktverantwortung nach den Absätzen I und 2 zu erfüllen haben. Dabei legt sie zugleich fest, für welche Erzeugnisse und in welcher Art die Produktverantwortung wahrzunchmen ist.

\section{I. Rechtspflicht der Produktverantwortung?}

Das anspruchsvolle Konzept der Produktverantwortung, so wie es $\$ 22$ Abs. x, 2 $\mathrm{KrW}-/ \mathrm{AbfG}$ formuliert, bedarf als Kernstück der neuen Abfallgesetzgebung zu seiner Wirksamkeit einer irgendwie gearteten rechtlichen Verbindlichkeit. Eine solche rechtliche Verbindlichkeit kommt den Zielsetzungen der Produktverantwortung in $\$ 22$ Abs. I, 2 KrW-/AbfG indessen nicht zu. Vielmehr handelt es sich um cine Norm mit bloßem Appellcharakter ${ }^{42}$. Die Gegenauffassung versteht die Regelung über die Produktverantwortung in $\$ 22$ Abs. I $\mathrm{KrW}-/ \mathrm{AbfG}$ als latente Grundpflicht der Produzenten. Anders als die Grundpflichten des Anlagenbetreibers nach Immissionsschutzrecht sei die Pflicht zur abfallrechtlichen Produktverantwortung zwar nicht unmittelbar verbindlich und erzwingbar; ihr könne jedoch für den Erlaß konkretisierender Rechtsverordnungen nach $\ 22$ Abs. 4, 23, $24 \mathrm{KrW}-/ \mathrm{AbfG}$ und hier insbesondere für deren Verschärfung besondere Bedeutung zukommen. Die latent vorhandene Grundpflicht der Produktverantwortung müsse lediglich über Rechtsverordnungen aktualisiert werden, um konkrete Rechtswirkungen zu entfal$\operatorname{ten}^{43}$. Die Produktverantwortlichen scien bereits jetzt gehalten, sich an den im Gesetz umschriebenen Anforderungen an die Produktverantwortung zu orientieren. Sie müßten sich deshalb auf Beeinträchtigungen oder Erschwernisse für ihrc Produktion und die Vermarktung ihrer Produkte einstellen. Ihr Vertrauen in eine gleichbleibende Rechtslage sei gemindert. Mit den jeweiligen Verordnungen würden deshalb lediglich bereits vorhandene Pflichten konkretisicrt. Der bei Erlaß der Verordnung zu beachtende Verhältnismäßigkeitsgrundsatz lasse angesichts der bereits bestehenden Grundpfliche zur Produktverantwortung ein größeres Maß an Beeinträchtigung für Hersteller und Vertreiber von Produkten als zumutbar erscheinen.

Diese Auffassung ist nicht überzeugend. Zunächst ist darauf hinzuweisen, daß konkrete Anforderungen an die Produktverantwortlichkeit sich aus $\$ 22$ Abs. I $\mathrm{KrW}-/ \mathrm{AbfG}$ für den Unternehmer nicht ergeben. Welche Pflichten zukünftig wel-

4I Nahere Erlauterungen dieser Konkretisierung bei Frenz, KrW-/AbfG (Fn. 10), \$22 Rdnr. 8 ff.; Hoffmann, in: Brandr/Ruchay/Weidemann, KrW-/AbfG (I'n. 7), \$2 2 Rdnr. 445 ff., Versteyl, in:Kunig/Paetow/Verstcyl, KrW-/AbfG (Fn. 14), $\$ 22$ Rdnr. i7 ff.

42 Wic her: Berg/Hosch, L lR 1997 (Fn. 32), 94; Becknlann, Produktverantwortung - Grundsatze und zulissigc Reichweite, UPR 1996, 4t, 45; ders., Psoduktbezogene Rucknahmeregelungen, in: Hoppe/ Baucr/I'aber/Schink (Hrsg.), Auswirkungen des Kreislaufwirtschafts- und Abfallgesetzes auf die offentlich-rechtichen Entsorgungstrager, 1996, S. is 5, is 6 f.; Fluck, KrW-/AbfG (Fn. 16), $\$ 22$ Rdnr. 64; Weidemann, Umweitschutz durch Abfallrechs, NVwZ 1995, 631, 634. A. A., namlich fur eine latente Grundpflicht: Frenz, KrW /AbfG (Fn. 10), \$22 Rdnr. s: Hoffmann, DVBl 1996 (Fn. 21), 900; ders., in: Brande/ Ruchay/Weidenann, KrW-/AbfG (Fn. 7), \$22 Rdnr. 26; Petersen/Rid, NJW 1996 (Fn.9), ro; Versteylf Wendenburg, 1)as neue Kreislaufwirtschafts- und Abfallgesetz, NVwZ 1994, 833, 839.

$43 \mathrm{Vgl}$. dazu Frenz, Hoffmann und Petersen/Rid, jeweils ebd. (Fn. 42). 
chen Adressaten der Produktverantwortung auferlegt werden könnten, ist aus $\ 22$ Abs. I KrW-/AbfG angesichts der vielfältigen Gestaltungsmöglichkeiten des Verordnungsgebers überhaupt nicht vorhersehbar. Eine Abschwächung des bei Erlaß von Verordnungen zu beachrenden Verhältnismäßigkeitsgrundsatzes kann deshalb allein wegen der Grundnorm über die Produktverantwortung in $\$ 22 \mathrm{Abs}$. I, $2 \mathrm{KrW}-1$ $\mathrm{AbfG}$ nicht eintreten ${ }^{44}$. Zudem ist darauf hinzuweisen, daß $\$ 22 \mathrm{KrW}$-/AbfG keinerlei Rechtsfolgen anordnet. In $\ 22 \mathrm{Abs.} 4 \mathrm{KrW}-/ \mathrm{AbfG}$ ist vielmehr ausdrücklich geregelt, daß es zur Umsetzung von einzelnen Anforderungen der Produktverantworllichkeit des Erlasses von Rechtsverordnungen nach $\$ \$ 23,24 \mathrm{KrW}-/ \mathrm{AbfG}$ bedarf. Eine Grundpflicht, die umgesetzt werden könnte, stellt die Regelung über die Produktverantwortung in $\$ 22 \mathrm{Abs}$. I KrW-/AbfG deshalb nicht dar. Auch latent ist eine Grundpflicht in dieser Norm nicht enthalten. Das zeigt der Regelungsmechanismus, der vom Gesetzgeber mit der Schaffung der Produktverantwortung in $\ 22$ Abs. I $\mathrm{KrW}-/ \mathrm{AbfG}$ verbunden war, überdeutlich: Aus $\ 22 \mathrm{Abs}$. I $\mathrm{KrW}-/ \mathrm{AbfG}$ läßt sich der Wille des Gesetzgebers entnehmen, die soziale Marktwirtschaft um eine ökologische Komponente, nämlich die Produktverantwortung des Unternehmers zu ergänzen. $\$ 22$ Abs. r KrW-/AbfG dient dabei als eine Norm, die eigenes Verhalten der Unternehmer in Richtung auf die Verwirklichung dieses Ziels anstoßen soll. Freiwilliges Verhalten soll, dies wird dem Unternehmer in $\ 22$ Abs. $4 \mathrm{KrW}-/ \mathrm{AbfG}$ signalisiert, Verordnungen, die konkrete rechtliche Anforderungen zur Umsetzung der Produktverantwortlichkeit enthalten, entbehrlich machen ${ }^{4 !}$. Über $\$ 22 \mathrm{Abs}$. I $\mathrm{KrW}-/ \mathrm{AbfG}$ appelliert der Geserzgeber lediglich an die Wirtschaft, den Gedanken der Produktverantwortung ernst zu nehmen. Er vcrbindet dies allerdings mit einer Drohgebärde, indem er zugleich auf die Möglichkeit des Erlasses von Rechtsverordnungen zur Konkretisierung der Produktverantwortung hinweist. Das Konzept des Gesetzgebers für Produktverantwortlichkeit beruht damit auf einem doppelstufigen Modell: Zunächst haben die Produktverantwortlichen Raum zur Eigeninitiative. Gelingt es hierüber nicht, Abfällc zu minimieren, den Schadstoffgehalt von Abfällen zu reduzieren und eine Wiederverwend- und Verwertbarkeit von Produkten zu schaffen, steht als zweite Stufe die Verordnungsermächtigung zur Verfügung, mit der der Gesetzgeber regelnd auf die Produktion und die Gestaltung von Produkten einwirken $\mathrm{kann}^{46}$. Genau dies ist auch der Weg, den der Gesetzgcber in der vergangenen Legislaturperiode beschritten hat: Sein Ziel war zunächst die freiwillige Verhaltensänderung. Zur Verhaltenssteuerung sollten dabei in erster Linie ebenfalls freiwillige Instrumente, nämlich Sclbstverpflichtungen der Wirtschaft, eingesetzt werden, die Vorrang vor Rechtsverordnungsermächrigungen haben sollten ${ }^{47}$. Erst wenn solche freiwilligen Lösungen scheitern, sollte überhaupt - und dann auch nur in einzelnen Fällen - von den Verordnungsermächtigungen Gebrauch gemacht werden ${ }^{48}$.

Auch in diesem Punkt zeigt sich, daß die wichtigsten neuen Leitlinien des KrW-/ AbfG keineswegs rechlliche Verbindlichkeiten beanspruchen, sondern lediglich Initialzündungen, Appelle zu freiwilligem Verhalten der Produzenten darstellen sollen. Das gilt auch und besonders für dic Produktverantwortung. Auch sie stelle deshalb in erster Linie einen symbolischen Akt dar, der über Rechtsverordnungen zwar kon-

44 Wie hier Beckmann, UPR 1996 (Fn. 42), 4 ; ders. in: Hoppe u. a., Auswarkungen des KrIW-/AbfG (Fn. 42), 157.

4s Vgl. ecwa Bender/Sparwasser/Enge!, Umwelerecht 1995, 10, Rdnr. 17; Berg/Hosch, Jb. UTR 1997 (Fn. 32), $94 \mathrm{f}$.

$46 \mathrm{Zu}$ diesem doppelstufigen Modell Berg/Hösch, Jb. UTR 1997 (Fn. 32), 88 f., $94 \mathrm{f}$.

47 So die Koalitionsvereinbarung zwischen CDU/CSU und FDP vom I 1. 11. 1994, Abschnit VI, Okologie und Markcwirtschaft, S. 35 .

$4^{8} \mathrm{Vgl}$. dazu A ntwort der Bundesregierung auf die Kleine Anfrage der Abgeordneten Lippold u. a., Vollzug des neuen Abfallrechts in Deutschland, BT-Drs. $13 / 8406$. 
kretisiert werden kann, aus sich heraus jedoch keinerlei Verbindlichkeit bean-

sprucht.

\subsection{Konkretisierung der Produktverantwortung durch freiwillige Vereinba- rungen und Rechtsverordnungen}

Zur Realisierung der Produktverantwortung sind bisher in der Bundesrepublik drei Rechtsverordnungen erlassen worden, nämlich

- die Altauto-Verordnung,

- die Verpackungs-Verordnung und

- die Batterieverordnung.

In der Diskussion ist darüber hinaus eine sogenannte IT-Geräte-Verordnung, durch die Pflichten für die Hersteller und Vertreiber der Geräte der Informations- und Kommunikationstechnik - sogenannten IT-Geräten - geregelt werden sollen ${ }^{49}$.

Bei näherem Hinsehen zeigt sich, daß in diesen Verordnungen zwar Elemente der Produktverantwortung der Hersteller und Vertreiber geregelt worden sind, daß der Verordnungsgeber sich jedoch gescheut hat, den Gedanken der Produktverantwortung wirklich ernst zu nehmen und den Herstellern und Vertreibern die volle Verantwortung für die Folgen des In-Verkehr-bringens ihrer Produkte aufzulegen:

Die Altauto-Verordnung regelt die Produktverantwortung der Hersteller von Automobilen nur ansatzweise. Sie basiert - wie erwähnt - auf der freiwilligen Selbstverpflichtungserklärung zur umweltgerechten Altautoverwertung. Nach dieser Selbstverpflichtungserklärung haben sich die Branchen der Automobilherstellung, des Handels, der Zuliefer- und Entsorgungswirtschaft verpflichtet,

- zum Aufbau einer flächendeckenden Infrastruktur zur Annahme und Verwertung von PKWs und Altteilen aus PKW-Reparaturen,

- zur umweltverträglichen Entnahme von Betriebsstoffen, Demontage und Verwertung von Teilen und Materialien von Altautos sowie zur ordnungsgemäßen Beseitigung der anfallenden nicht verwertbaren Abfälle,

- zur Verbesserung der Verwertung von Altautos, indem bis spätestens zum Jahre 2002 die zu beseitigenden Abfälle von heute durchschnittlich 25 Gewichtsprozent des Altautos im Durchschnitt pro Fahrzeughersteller auf maximal is $\%$ und bis zum Jahre zors auf maximal s Gewichtsprozent verringert werden,

- die Verwertungseigenschaften von Fahrzeugen im Rahmen der Produktverantwortung kontinuierlich zu verbessern,

- generell Altautos ihrer Marke vom letzten Halter zu marktüblichen Konditionen über die dazu vom Hersteller genannten Stellen zurückzunehmen und

- zukünftig zugelassene Fahrzeuge unter den Voraussetzungen kostenlos zurückzunehmen, daß der jeweilige PKW für den Markt der EU bestimmt war, nicht älter als zwölf Jahre ist, zuletzt mindestens sechs Monate in Deutschland auf den Anlieferer zugelassen gewesen ist, der PKW vollständig rollfähig und frei von Abfällen ist und keine wesentlichen Beschädigungen aufweist.

Diese Selbstverpflichtungserklärung ist durch die Altautoverordnung lediglich ergänzt worden; sie soll die kooperative Lösung über die Selbstverpflichtungserklärung vervollständigen und weitergehende abfallwirtschaftliche Ziele außerhalb der Selbst-

$49 \mathrm{Zu}$ diesen Regelungen vgl. den Überblick bei Beckmann, in: Hoppe u. a., Auswirkungen des KrW./AbfG (Fn. 42), S. 167 ft.; zur Altautoverordnung: Faber, Altautoentsorgung: Umweltschutz und Wettberwerb, UPR 1997,43 iff. 
verpflichtung erreichen ${ }^{\circ}$. Der Anhang der Altauto-Verordnung setzt dabei Standards für die Wiederverwendung, Verwertung und Beseitigung der Abfälle, die aus demontierten Altautos gewonnen worden sind. Sie sind hiernach vorrangig einer Wiederverwendung oder Verwertung zuzuführen, und zwar in größtmöglichem Umfang (Ziffer 3.2.4. I Anhang AltautoV). Der Umfang der zu beseitigenden Abfallmenge von bis zum Jahre 2002 durchschnittlich is Gewichtsprozent und ab dem Jahre 20 rs durchschnittlich fünf Gewichtsprozent ist ebenfalls festgeschrieben worden (Ziffer 4.I.2 AltautoV). Wie diese Ziele umgesetzı werden, überläßt die AltautoV ausschließlich der produzierenden Automobilindustrie. Die recyclinggerechre Konstruktion von Kraftfahrzeugen wird keiner Regelung zugeführt. Ähnliches gilt für den Schadstoffgehalt von Materialien, die in Automobilen Verwendung finden. Auch insoweit wird über die Altauto-Verordnung ebensowenig wie über die Selbstverpflichtungserklärung der Automobilindustrie Einfluß genommen. Damit wird es der Automobilindustrie überlassen, wie das festgeschriebene Zicl der Wiederverwendung bzw. Verwertung von $85 \%$ des in Autos eingesetzren Materials bis zum Jahre 2002 und von $95 \%$ bis zum Jahre 2015 erreicht wird. Diese Lösung dürfte insgesamt sachgerecht sein, denn es ist kaum möglich, über eine Verordnung zur Produktverantwortung lenkend auf die recyclinggerechte Konstruktion von Altaucos Einfluß zu nehmen. Problematisch ist allerdings, daß hinsichtlich des Schadstoffanteils weder in der freiwilligen Selbstverpflichtungserklärung noch in der Altauto-Verordnung irgendwelche Vorgaben gemache sind. Eine Anreicherung von Schadscoffen im Wirtschaftskreislauf läßt sich auf diese Weise kaum wirksam verhindern. Besonders problematisch ist im übrigen, daß die koscenlose Rücknahme von Altfahrzeugen lediglich solche Automobile betrifft, die nach Inkraftreten der Alcauto-Verordnung in den Verkehr gebracht und höchstens zwölf Jahre alt sind. Damic werden die heute auf dem Markt befindlichen Automobile vom Rücknahme- und Verwertungssystem weitgehend ausgeschlossen. Jcdenfalls eine Kostenverantwortliehkeit der Hersteller und Händler für das Recycling dieser Automobile wird nicht anerkannt. Dies ist kaum eine Lösung, die dem Gedanken der Produktverantwortlichkeit wirklich Rechnung trägt.

Eine volle Kostenverantwortlichkeit ist auch den Herstellern und Vertreibcrn von Batterien durch die Batterie-Verordnung nicht auferlegt worden. Auch diese Regelung basiert auf dem Konzept, so wenig wie möglich auf den Produktionsprozeß einzuwirken und es den Herscellern und Vertreibern selbst zu überlassen, ein Rücknahmesystem mit dem Ziel der größtmögliehen Verwercung von Batterien zu etablieren. Im Unterschied etwa zur Altauto-Verordnung enthält dabei die BattV zwarals Umsetzung entsprechender EG-rechtlicher Vorgaben - Verkehrsverbote für bestimmte Batterien; anders als in der Altauto-Verordnung sind jedoch Rücklauf- und Verwercungsquocen in der BattV nicht enthalcen. Schon deshalb bleibr die Regelung der Produktverantwortung in der BattV unvollkommen. Das gilt insbesondere auch für die Kostenzurechnung: Soweic es um Batterien aus privaten Haushaltungen und Kleingewerbe geht, ist den öffentlich-rechrlichen Entsorgungsträgern nach $₫ 9$ Abs. 2 BattV die Aufgabe übertragen, die von ihnen erfaßten Batterien einem Rücknahmesystem der Hersteller zur Abholung unentgeltlich bereitzustellen. Die Kosten für die Erfassung von Altbatterien werden damit den öffentlich-rechtlichen Gebietskörperschaften auferlegt - dem Gedanken der Produktverantwortung entspricht dies keineswegs. Folge davon ist, daß das inzwischen ecablierce gemeinsame Rücknahmesystem Batterien zwar mehr als 100000 Übergabestellen für den privaten $\mathrm{Hande]}$

so Vgl. Begrundung zur Altauto-Verordnung vom 30. 5. 1997, BT-Drs. 13/7780, S. 2; Faber, UPR I997 (Fn. 49), 832 . 
einrichten will, für die kommunalen Gebietskörperschaften es jedoch bei lediglich 450 Übergabestellen belassen möchte. Auf diese Weise wird die Produktverantwortlichkeit in kostenmäßiger Hinsicht zu Lasten der kommunalen Gebietskörperschaft und damit letztlich des Gebührenzahlers - ausgehöhlt.

Die Verpackungsverordnung als Muster einer Regelung der Produktverantwortung überläßt es ebenfalls den Herstellern und Vertrcibern von Verpackungen, die Entscheidung darüber zu treffen, wie die Anforderungen der Verpackungsverordnung im einzelnen umgesetzt werden sollen. Das gilt sow ohl für die Vermeidung von Verpakkungen, ihre Zusammensetzung (abgesehen von den Verkehrsbeschränkungen des $\$ r_{3}$ VerpackV) als auch für die Organisation von Wiederverwertungssystemen im Sinne des $\$ 6$ Abs. 3 VerpackV. Der Ansatz ist damit derselbe wie der, der der Altautoverordnung und der Batterieverordnung zugrunde liegt: Über die Verpflichtung zur Rücknahme verbrauchter Produkte sollen marktwirtschaftliche Anreize dafür geschaffen werden, die Wicderverwendung und Wiederverwertung im größtmöglichen Umfang durchzuführen. Gerade was diesen Punkt angeht, stellt die Novelle der VexpackV freilich einen Rückschritt gegenüber dem bisherigen Recht dar: Die stoffliche Wiederverwertung von Kunststoffverpackungen ist - anders als früher - nicht mehr in vollem Umfang geboten. Zwar müssen nach Ziffer 1.2 des Anhangs I zu $\$ 6$ VerpackV Kunststoffverpackungen ab dem I. I. I996 in einer Größenordnung von 50\% und ab dem I. I. 1999 in einer Größenordnung von $60 \%$ einer Verwertung zugeführt werden. In Gegensatz zu früher bezieht sich die Verwertungsquote dabei allerdings nicht mehr auf alle während eines Jahres in Verkehr gebrachten Verkaufsverpackungen, sondern lediglich auf jene, die von Herstellern und Vertreibern in den Verkehr gebracht worden sind, die sich an einem Rücknahmesystem nach $\$ 6$ Abs. 3 VerpackV, also dem Dualen System beteiligen. Hinzu kommt, daß - in Umsetzung der Anforderungen der EG-Verpackungsrichtlinie $^{51}$ - nur mehr $60 \%$ der zu verwertenden Kunststoffverpackungen werkstofflich verwertet werden müssen; der Rest kann auch thermisch verwertet werden. In der Praxis wird dies bedeuten, daß die werkstoffliche Verwertung von Kunststoffverpakkungen deutlich reduziert werden wird und - angesichts der derzeitigen Praxis, in Müllverbrennungsanlagen Kunststoffabfälle als Abfälle zur thermischen Verwertung anzunehmen ${ }^{52}$ - voraussichtlich in Müllverbrennungsanlagen zur Verwertung thermisch behandelt werden. Ob dies wirklich der Produktverantwortung entspricht, mag man bezweifeln. Wirtschaftlich betrachtet ist diese Lösung sicherlich sinnvoll. Unter ökologischen Aspekten bleiben jedoch an der Sinnhaftigkeit dieser Regelung erhebliche Fragestellungen.

\subsection{Produktverantwortung - ein Papiertiger?}

Bei näherer Betrachtung zeigt sich damit, daß die Produktverantwortung als wesentliche Neuerung des $\mathrm{KrW}-/ \mathrm{AbfG}$ nicht viel mehr als ein Papiertiger ist. Die an alle Produzenten gerichteten Regelungen des $\$ 22 \mathrm{KrW}$-/AbfG wirken lediglich als Appell, sind rechtlich indessen nicht vollziehbar. Verordnungen zur Regelung der Produktverantwortlichkeit gibt es derzeit lediglich in einigen Bereichen. Diese Verordnungen setzen den Gedanken der Produktverantwortung aber nicht in Reinform

SI Dazu Schink, in: Rengeling, HEDUR, $\$ 74$, Rdnrn. 7 ff.

52 Zu dieser Praxis vgl. OVG NW, Beschl. v. 25.6. $1998-20$ B 1424/97-; Nds. OVG, Beschl. v. 6. 5. 1998-7 M 3055/97-; Schink, Kommunale Abfallentsorgung im Wettbewerb mit der Entsorgungswirtschaft, Der Landkreis $1998,53,57 \mathrm{f}$. 
um, sondern verschieben Kostenverantwortlichkeiten zugunsten anderer, nämlich der öffentlichen Hand (BattV) oder der Endverbraucher (AltautoV), oder eröffnen im Sinne einer kostenminimierenden Lösung ökologisch zweifelhafte Verwertungswege. Auch die Produktverantwortung als wesentliches Ziel des neuen Abfallrechts trägt damit in großen Bereichen symbolhafte Züge. Als Drohgebärde mag dabei die Rechtsverordnungsermächtigung zur Konkretisierung der Produktverantwortung in manchen Fällen in der gewerblichen Wirtschaft durchaus Wirkungen haben. Besonders groß sind diese indessen nicht. Auch hier zeigt sich vielmehr, daß Wirtschaftlichkeitsüberlegungen in erster Linie zu einem Ernstnehmen der Produktverantwortlichkeit führen. Allerdings: Konkrete Regelungen, dic in die Produktion eingreifen, kann und sollte der Gesetz- und Verordnungsgeber in diesem Bereich nicht treffen. Sinnvoll ist es aber, auf freiwillige Selbstverpflichtungen, ergänzt durch Verordnungen zu setzen. Die Altautoverordnung und die sie begleitende freiwillige Selbstverpflichtungserklärung können dabei durchaus vorbildhaft sein, wenn ein Gesichtspunkt in der Zukunft stärker als bislang berücksichtigt wird: Die Produktverantwortung muß wirklich ernst genommen werden; freiwillige Selbstverpflichtungen müssen der Produktverantwortung wirklich gerecht werden und die Probleme, die bewältigt werden sollen, wirklich lösen.

\section{Mebrprivate Verantwortung für die Entsorgung statt offentlicher Daseins- vorsorge}

Als wesentliche Neuerung, ja als Paradigmenwechsel im Abfallrecht wird von viclen die Neuregelung der Entsorgungsverantwortlichkeit durch das $\mathrm{KrW}$-/AbfG angesehen ${ }^{33}$. Die $\int \mathbb{S} S$ Abs. 2 und ro Abs. I KrW-/AbfG weisen die Vcrantwortung für die Verwertung und Beseitigung von Abfällen den Abfallerzeugern und -besitzern zu. Die öffentlich-rechtlichen Entsorgungsträger sollen in eine Reservezuständigkeit für die Bereiche gedrängt werden, in denen eine marktwirtschaftliche Orientierung des abfallwirtschaftlichen Geschehens nicht möglich ist. Zur Begründung wird darauf hingewiesen, daß das Festhalten an überkommenen Entsorgungsstrukturen, verbunden mit entsprechenden Überlassungs- und Andienungspflichten, beim Aufbau einer Kreislaufwirtschaft mit dem Ziel der Abfallvermcidung kontraproduktiv sei; Kreislaufwirtschaft und öffentliche Entsorgung schlössen sich tendenziell aus. Natürliche Träger der Stoffkreisläufe seien die Unternehmen und Gewerbebetriebe, nicht jedoch der Staat oder die entsorgungspflichtigen Körperschaften ${ }^{54}$. Die Aufgaben der öffentlich-rechtlichen Entsorgungsträger müßten, solle der Gedanke der Kreislaufwirtschaft wirklich ernst genommen werden, zunehmend eingegrenzt und auf die immer wcniger bedeutende Aufgabe der Abfallbeseitigung und des Einsammelns der Abfälle zur Beseitigung beschränkt werden"s. Die Erkenntnis und das Wissen um die Mißerfolge der öffentlichen Abfallentsorgung in der Vergangenheit hätten den Gesctzgeber zu einer Neudefinition der Rollenverteilung zwischen öffentlicher und privater Entsorgung veranlaßt. Der Vorrang der öffentlichen Entsorgung sei zugunsten einer verursacherbezogenen Verantwortlichkeit von Abfallbesitzern und Abfallerzeugern aufgegeben worden, und zwar deshalb, damit bereits bei der Produktion vom Abfall her gedacht werden könne ${ }^{66}$.

(3) Nachweise dazu schon oben Fn. 10.

s4 So: Beckmann, UPR 1996 (Fn. 42), 41; Weidemann, NVwZ r99s (Fn. 42), 632

ss So: Beckmann, ebd. (Fn. 54), 41 .

s6 Beckmann, ebd. (Fn. s4). 
Bei näherer Betrachtung der Mechanismen der Privatisierung der Entsorgungsverantwortlichkeit zeigt sich, daß abgesehen von den Leitnormen der $\$ \$ s$ Abs. 2 und ro Abs. I KrW-/AbfG die Regelung der Entsorgungszuständigkeit überhaupt nicht geändert worden ist, sondern die eigentliche Neuerung darin liegt, daß - ausgelöst zum einen durch dic Privatisicrungsphilosophie des $\mathrm{KrW}-/ \mathrm{AbfG}$ und zum anderen durch eine stärkere Kostenorientierung der Produzenten und Abfallbesitzer - die Verschiebung der Abfallströme über eine Intensivierung der Verwertung von Abfällen durch gewerbliche Unternehmen erfolgt ist.

Regelungen über die Entsorgungszuständigkeiten ergeben sich nicht alleine aus $\$ \$ S$ Abs. 2 und to Abs. I KrW-/AbfG. Diese Bestimmungen stehen vielmehr in engem Zusammenhang mit den Regelungen über die Überlassungspflichten in $\$_{13}$ Abs. I $\mathrm{KrW}-/ \mathrm{AbfG}$. Durch diese Regelung sind dic alten Entsorgungszuständigkeiten der öffentlichen Hand quasi durch die Hintertür wieder eingeführt worden: Für Abfälle zur Beseitigung sind insgesamt die kommunalen Gebietskörperschaften entsorgungspflichtig - sieht man einmal von den kaum praktischen Einschränkungen für die Bescitigung gewerblicher Abfälle in eigenen Anlagen ( $\$ 1_{3}$ Abs. I Satz $2 \mathrm{KrW}-/ \mathrm{AbfG}$ ) ab. Für die Verwertung von Abfällen gibr es cbenfalls eine Zuständigkeit der kommunalen Gebietskörperschaften. Diese beschränkt sich allerdings auf die Verwertung von Abfällen aus privaten Haushaltungen. Für Abfälle zur Verwertung aus anderen Herkunfrsbereichen und damit insbesondere für Abfälle zur Verwertung aus dem Herkunftsbereich gewerblicher Unternehmen gibt es hingegen keine Überlassungspflicht an die öffentlich-rechtlichen Gebietskörperschaften ${ }^{57}$. Rechtlich hat sich damit an den Entsorgungsverantwortlichkeiten eigentlich gar nichts geändert: Nach wie vor sind die kommunalen Gebietskörperschaften für die Abfallbeseitigung zuständig. Dic Verwertung von Abfällen aus anderen Herkunftsbereichen als privaten Haushaltungen gehörte auch bislang schon nicht zu ihren Aufgaben. Diese waren vielmehr als sogenannte Wertstoffe aus dem Abfallbegriff des $\$ 1$ Abs. I AbfG herausdefiniert und unterfielen damit nicht dem Anwendungsbereich des Abfallrechts. Die eigentliche Neuerung des KrW-/AbfG besteht deshalb nicht so sehr darin, daß die Entsorgungszuständigkeiten neu verteilt worden sind, sondern in der Einbeziehung der bisherigen Wertstoffe als Abfälle zur Verwertung im Sinne der $\$ \llbracket 3$ Abs. I Sarz 2, 4 Abs. $3,4 \mathrm{KrW}-/ \mathrm{AbfG}$ in den Anwendungsbereich des Abfallrechts und in der Sehaffung einer Verpflichtung für gewerbliche Unternehmen, diese Abfälle zu verwerten, wenn die Voraussetzungen des $\$$ s Abs. 4, s KrW-/AbfG vorliegen. Von einem wirklichen Paradigmenwechsel weg von der öffentlichen Daseinsvorsorge in der Abfallbeseitigung hin zu mehr privater Verantwortlichkeit kann deshalb keine Rede sein. Auch die Bestimmungen der $\mathbb{S} S$ s Abs. 2, I 1 Abs. I KrW-/AbfG haben einen eher symbolischen Charakter, ändern aber an der Entsorgungsverantwortung wegen der Bestimmung über die Überlassungspflichten in $\$ r_{3}$ Abs. I KrW-/AbfG im Grunde nichts.

57 Einzelheiten zu diesen Regelungen bei Queitsch, Das neue Kreislaufwirtschafts- und Abfallgeset\%, UPR 1995, 413 ff.; Petersen/Rid, NJW 1995 (Fn. 9), 8 f.; Schink, Offentliche und private Entsorgung, NVwZ 1997, 435, 436ff, und in den Kommentierungen von Frenz, Fluck, Kunig/Paetow/Versteyl und Weidemann (in: Brande/Ruchay/Weidemann), jeweils zu $\S_{13} \mathrm{KrW}$-/AbfG. 


\subsection{Feblende Trennschärfe zwischen Abfällen zur Verwertung und Abfällen zur Beseitigung}

Dic eigentliche Trennlinie zwischen privater und öffentlicher Entsorgung wird im Ergebnis durch die Regelungen über die Abgrenzung zwischen Abfällen zur Verwertung einerseits und Abfällen zur Beseitigung andererseits getroffen. Auch insoweit ist allerdings festzustcllen, daß die Trennlinien, die das KrW-/AbfG dazu gibt, alles anderc als klar sind, mit der Folge, daß nicht nach rechtlichen oder ökologischen Kriterien entschieden wird, ob ein Abfall cin Abfall zur Verwertung ist, sondern daß insoweit vor allem Kostenüberlegungen maßgebend sind:

$\$ 3$ Abs. I Satz $2 \mathrm{KrW}$-/AbfG definiert Abfälle zur Verwertung dahin, daß dies Abfälle sind, die verwertet werden; Abfälle zur Beseitigung sind nach dicscr Norm dengegenüber Abfälle, die nicht verwertet werdern. $\mathrm{Da}$ damit eine wirkliche Abgrenzung zwischen Abfällen zur Verwertung und Abfällen zur Beseitigung nicht getroffen werden kann, liegt auf der Hand. Darauf ziclt dic Norm indessen auch nicht ab. Sie stellt vielmehr klar, daß es für die Abgrenzung zwischen Abfallverwertung und Abfallbeseitigung auf das tatsächliche Gcschehen, also darauf ankommt, welcher Behandlung ein Abfall im einzelnen unterzogen wird's ${ }^{8}$. Die Folge sind crhebliche Schwierigkeiten bei der Klassifizierung von Abfällen als Abfälle zur Beseitigung und Abfälle zur Verwertung: Nicht selten ist einer Verwertung von Abfällen einc Behandlung in einer Sortieranlage vorgeschaltet. Eine Sortierung kann aber sowohl einer Verwertung als auch als Vorbehandlungsschritt der Beseitigung zuzuordnen sein. Die Klassifizierung eines Abfalls zur Verwertung setzt damit gerade nicht voraus, daß Abfall so anfällt, daß er ohne weiteres einer Verwertung zugeführt werden kann. Auch gemischte Abfälle, denen man manchmal ihre Verwcrtungseignung überhaupt nicht ansieht und die in der Vergangenheit als Abfälle zur Beseitigung den Anlagen der öffentlich-rechtlichen Gebietskörperschaften zugeführt worden sind, können hiernach Abfälle zur Verwertung darstellen. Die Inhomogenität eines Abfallgenisches spielt damit für die Klassifizierung als Abfall zur Verwertung oder zur Beseitigung keine wesentlichc Rolle. Entscheidend ist viclmehr nach $\$ 4$ Abs. 3, $4 \mathrm{KrW}$-/AbfG sowohl für die Abgrenzung der stofflichen Verwertung von der Bescitigung als auch für die der energetischen Verwertung von der thermischen Beseitigung die Frage, wo der Hauptzweck der Maßnahme bei eincr wirtschaftlichen Betrachtung liegts9.

Auch dieses Abgrenzungskriterium hat sich freilich bislang in seiner praktischen Anwendung als ehcr problematisch erwiesen. So ist etwa in der verwaltungsgerichtlichen Rechtsprechung angenommen worden, daß die Nutzung des Volumens eines Stoffes, also seiner Fähigkeit, einen Raum zu füllcn, für das Vorliegen einer Abfallverwertungshandlung ausreicht, wenn dieser $Z$ weck über dic bloße Bescitigung hinaus als Hauptzweck erreicht werden soll ${ }^{60}$. In der Sache ging es dabei um die Verwendung von Kunststoffgranulat aus DSD-Sammlungen zum Bergversatz. Da jeder Abfall raumfüllende Eigenschaften hat, ist es zumindest zweifelhaft, ob die

58 Kunig, in: Kunig/Paetow/Versteyl, KrW-/AbfG (Fn. r4), $\$ 3$ Rdnr. 26; Kix, Auswirkungen des Kreislaufwirtschaf(s- und Abfallgesetzes auf dic Satzungen der öffentlich-rechthchen Entsorgungstrager, in: Hoppe u. a., Auswirkungen des KrW-/AbfG (Fn.42), 191, 196; Frenz, KrW-/AbfG (Fn. 10), \$3 Rdnr. 28. Vgl. aber auch Weidemann, GewArch 1997, 311, 340, der diese Regelung als wahrhaft nsibyllinisch" und „für die Rechtsanwendung praktisch wertlos « kennzeichnet.

s9 Dazu: Dolde/Vetter, Abgrenzung von Abfallverwertung und Abfallbeseitigung nach dem Kreislaufwirtschatts- und Abfallgcsctz, NVwZ 1997, 937, 939 ff; Kunig, Der Abfallbegriff, NVwZ 1997, 209, 214 Schink, Der neue Abfallbegriff und seine Folgen, VerwArch 1997, 230, 2 50 ff.

60 VG Stuttgart, Lirc. v. 26. 12. 1996-14 K 3580/95 -, in: Brandu/Ruchay/Weidemann, KrW-/AbfG (Fn. 7), $100 \mathrm{C}, \Omega_{4} \mathrm{KrW}-/ \mathrm{AbfG}, \mathrm{Nr} .2$. 
gefundene Abgrenzung wirklich eine Abgrenzung darstellt oder nicht alle cinlädt, über die raumfüllenden Eigenschaften des Abfalls Abfälle als Abfällc zur Vcrwertung in Abgrabungen unter anderen Umwelt - und damit Kostenbedingungen zu verbringen als diese bei der Verbringung auf eine Deponie üblich sind.

Zur näheren Abgrenzung der Abfälle zur Verwertung von Abfällen zur Beseitigung ist von der Länderarbeitsgemeinschaft Abfall (LAGA) das Hauptzweckkriterium ursprünglich dahingehend interpretiert worden, daß eine Verwertung jedenfalls dann gegeben ist, wenn mehr als so\% der Abfälle einer Verwertung zugeführt werden ${ }^{61}$. Auch diese Abgrenzung hat bislang jedenfalls in der Rechtsprechung keinen Bestand gehabt. So hat das VG Düsseldorf ${ }^{62}$ darauf hingewiesen, daß quantitative Betrachtungen für die Abgrenzung zwischen stofflicher Verwertung und Beseitigung nicht maßgebend seien. Verwertung im Sinne des $\mathrm{KrW}$-/AbfG bedeute vielmehr lediglich, daß ein konkreter wirtschaftlicher oder sonstiger Nutzen aus den Eigenschaften des Stoffes gezogen wird. Dies folge aus der Intention des Gesetzgebers, daß Abfälle in erster Linie verwertet werden müßten und erst nachrangig zu beseitigen sind. Dieser These des VG Düsseldorf wird man zwar sicherlich zustimmen können. Im Ergebnis läuft diese Rechtsprechung indessen darauf hinaus, daß es wirkliche Abgrenzungskriterien für Abfälle zur Beseitigung und Abfälle zur Verwertung in der Praxis jedenfalls nicht gibr. Vielmehr entscheiden allein wirtschaftliche Kriterien darüber, ob Abfälle in die Verwertung oder in die Beseitigung gehen. Die Entsorgungspraxis belegt dies überdeutlich: Dort, wo Entsorgungspreise in den Anlagen der öffentlichrechtlichen Gebietskörperschaften bcsonders hoch sind - so werden etwa im Kreis Wesel für die Verbrennung der Tonne Abfall mehr als 730,-DM vcrlangt - sind die zur Beseitigung angelieferten Abfälle aus gewerblichen Herkunftsbereichen nahezu auf Null zurückgegangen. In den Gebietskörperschaften jedoch, wo die Entsorgungskosten noch schr niedrig liegen - im Kreis Siegen-Wittgenstein beträgt der Preis für die Deponierung einer Tonne Abfall kaum mehr als so,- DM -, wird hingegen über einen Rückgang der Abfälle zur Beseitigung aus gewerblichen Herkunftsbereichen nicht geklagt. Im Gegentcil: Dort haben viele private Entsorgungsunternehmen Sortieranlagen gebaut mit dem Zicl, dort eine Sortierung als Teilaspekt einer Verwertungshandlung durchzuführen, da dann die Abfälle zur Beseitigung, die am Ende des Sortiervorgangs anfallen, gemäß $\$ 3$ Abs. s KrW-/AbfG der Gebietskörperschaft zu überlassen sind, in deren Bereich eine Sortieranlage gelegen ist. Gerade diese Praxis ermöglicht es, nahezu jedweden Abfall zu einem Abfall zur Verwertung zu deklarieren, in einer Sortieranlage verwertbare Bestandteile auszuschleusen, letztlich mir $\mathrm{dcm}$ Ziel, die kostengünstigste Beseitigungsart zu erschließen. Letztlich ist dies allerdings eine Folge der unklaren und in der Praxis nicht möglichen Unterscheidung von Abfällen zur Verwertung und Abfällen zur Beseitigung über die in $\$ 4$ Abs. 3, 4 $\mathrm{KrW}-/ \mathrm{AbfG}$ genannten Kritericn. Fin Wildwuchs in der Entsorgungslandschaft und ein Kampf um Abfälle ist die Folge. Bildlich gesprochen sucht sich der Abfall den Weg in das billigste Loch. Dies hat eine Verschiebung der Entsorgung weg von den

6r Vgl. Definition und Abgrenzung von Abfallverwertung und Abfallbeseitigung sowie von Abfall und Produkt nach dem Kreislaufwirtschafts- und Abfallgesetz - KrW-/AbfG in der am 17./18. 3. 1997 von der LAGA beschlossenen Fassung, Ziff. 5. 2. I »Mengenbetrachtung*, wo es heißt: „Eine Maßnahme ist dern Hauptzweek grundsátzlich dann als stoffliche Verwertung anzusehen, wenn die Bestandteile des Abfalls vollstandig oder zu einem uberwiegenden Teil einer erneuten Nutzung zugefuhrt werden. Ist der Anteil der gewonnenen oder genutzten Stoffe dagegen im Verhältnis zur Ausgangsmenge nur gering, so sind dic Ververtungseffekte im allgemeinen untergeordneter Nebenzweck einer in der Hauptsache auf Beseitigung des nichtverwertbaren Abfallanteils gerichteten Maßnahme. Als A nhalt hierfür kann ein Verwertungsanteil von weniger als die Halfte der Ausgangsmenge angenommen werdenx, abgedruckt bei Brandt/ Ruchay/Weidemann, K. W-/AbfG (Fn. 7), D roo.

62 Beschl. v. II. 3. 1997-17 L 12 16/97 -, abgedruckt bei Brande/Ruchay/Wiedemann, KrW-/AbfG (Fn. 7), rooc, $\$ 4 \mathrm{KrV}-/ \mathrm{AbfG}, \mathrm{Nr}$. I. 
öffentlich-rechtlichen Gebietskörperschaften hin zu privaten Entsorgungsunternehmen zur Folge gehabt. Ob damit allerdings die vom Gesetzgeber angestrebte Quantifizierung und Qualifizierung der Verwertung wirklich verbunden ist, daran bestehen ganz erhebliche Zweifel. In der Praxis findet wohl in vielen Fällen über eine "Verwertung" aus finanziellen und wirtschaftlichen Gründen eher eine Umgchung der Überlassungspflichten aus $\$ 13$ Abs. I $\mathrm{KrW}-/ \mathrm{AbfG}$ mit dem Ziel statt, bei der Entsorgung Geld zu sparen.

\subsection{Schadlosigkeit und Hochwertigkeit der Verwertung als Korrektiv?}

Die Schadlosigkeit der Verwertung, wie sie in $\$$ s Abs. 3 Satz I, 3 KrW-/AbfG mit dem Ziel gefordert wird, keine Schadstoffanreicherung im Wertstoffkreislauf zu verursachen, findet dabei in der Regel keine Beachtung ${ }^{63}$. Dieses Kriterium, dem für die Zulässigkeit der Verwertung selbständige Bedeutung zukomm ${ }^{64}$ und das sich sowohl auf dic Art der Verwertung als auch auf das aus ihr entstehende Produkt bezieht ${ }^{6 s}$, ist zwar sicher kein Mittel, Abfälle mit dem Ziel einer Auslastung von Leerständen in öffentlichen Beseitigungsanlagen in diese umzulenken ${ }^{66}$. Es soll jedoch die Qualität der Verwertung steucrn, eine Aufgabe, die dieses Tarbestandsmerkmal bislang allerdings nicht hat leisten können.

Ähnliches gilt für die Forderung in $\ S$ Abs. 2 Satz $3 \mathrm{KrW}-/ \mathrm{AbfG}$, eine der Art und Beschaffenheit des Abfalls entsprechende hochwertige Verwertung anzustreben. Damit ist gemeint, daß bei der Verwertung ein sogenanntes Downcycling nicht stattfinden soll, sondern daß der Verwertungserfolg nach Möglichkeit darin bestehen soll, Abfälle im Sinne ihrer ursprünglichen Z Zwecksetzung zu nutzen oder dic stofflichen Eigenschaften der zu verwertenden Materialien im Sinne einer Substitution von Primärrohstoffen einzusetzen ${ }^{67}$. Freilich: Auch diese Forderung stellt ein bloßer Appell an Abfallerzeuger und -besitzer dar. Verbindlichkeit für den Adressaten hat sic indessen nicht; konkrete Rechtspflichten können aus dem Postulat der Hochwertigkeit nicht abgeleitet werden, denn eine gesetzliche Vorschrift, die nur verlangt, daß ein bestimmter Erfolg anzustreben ist, schafft keine konkrete, unmittelbare Rechtspflicht ${ }^{68}$. Auch die Regelungen des KrW-/AbfG zur Lenkung der Qualität der Verwertung erweisen sich damit als nicht praktikabel und letztlich bloß symbolhafte Regelungen.

63 Zum Gebot der Schadlosigkeit bei der Verwertung vgl. etwa Ku:Iig, in: Kunig/Paetow/Versteyl, KrW-/ AbfG (Fn. 14), $\$$ S Rdnr. 28; Spocrr, in: Brandt/Ruchay/Weidemann, KrW-/AbfG (Fn. 7), $\$$, Rdnr. 8 gff.; Wcidemann, Anforderungen an die Kreislaufwirtschaft, in: Hoppe u. a., Auswirkungen des KrW-/AbfG (Fn. 42), S. 49, 60; Schink; Der neue Abfallbegriff und seine Folgen, VerwArch 1997, 230, 263; Frenz, $\mathrm{KrW}-/ \mathrm{AbfG}$ (Fn. to), \$s Rdnr. $22 \mathrm{ff}$

64 Vgl. nur Kunig, in: Kunig/Pactorw/Versteyl, KrW-/AbfG (Fn. I4), §s Rdnr. 28; Schink, VerwArch 1997 (Fn. 63), 263.

6s Vgl. Petersen/Rid, NJW 1995 (Fn. 9), 11

66 So mit Recht: Spocrr, in: Brande/Ruchay/Weidemann, KrW-/AbfG (Fn. 7), \$s Rdnr. 88; Weidemann, in: Hoppe u. a., Auswirkungen des KrW-/AbfG (Fn.42), S. 60.

67 In diesem Sinne Kunig, in: Kunig/Paetow/Versteyl KrW-/AbfG (Fn. 14), \$s Rdnr. ${ }_{3}$; Schink; VerwArch 1997 (Fn. 63), 262; Weidemann, NVwZ y995 (Fn. 42), 637; ders., in: Brandi/Ruchay/Weidemann, Kny-l AbfG (Fn. 7), \$s Rdnr. 49 .

68 Überwiegende Meinung vgl. erwa Kunig, in: Kunig/Pactow/Verstcyl, KrW-/AbfG (Fn. 14), \$s Rdnr. 14; Fluck, KrW-/AbfG (Fn. I6), \$s Rdnr. 116f.; Fren», KrW-/AbfG (Fn. 10), \$s Rdnr. 16; Schink, ZG 1996 (Fn. 22), ro7; Weidemann, NVwZ i 995 (Fn. 42), 637; ders., in: Brandr/Ruchay/Wcidemann, KrW-/AbfG (Fn. 7), \$s Rdnr. so. 
Das gilt auch für die Forderung des $\$ s$ Abs. $5 \mathrm{KrW}-/ \mathrm{AbfG}$, die Beseitigung zu wählen, wenn dies unter Berücksichtigung der zu erwartenden Emissionen, dem Ziel der Schonung der natürlichen Ressourcen, der einzusetzenden oder zu gewinnenden Energie und dem der Problematik der Schadstoffanreicherung in Erzeugnissen oder aus Abfällen gewonnenen Erzeugnissen die umweltverträglichere Lösung darstellt. Diese Regelung enthält nach ganz überwiegender Auffassung auch dann, wenn die Beseitigung bei einem ökologischen Folgenvergleich die umweltverträglichere Lösung darstellt, keine Beseitigungspflicht des Abfallerzeugers und -besitzers; aus dem Wortlaut und dem systematischen Zusammenhang mit $\$$ s Abs. $2 \mathrm{KrW}-/$ $\mathrm{AbfG}$ wird vielmehr gefolgert, daß der Verantwortliche in diesem Fall über ein Wahlrecht verfügt ${ }^{69}$.

\section{4. Keine verläßliche Abgrenzung zwischen privater und öffentlicher Ent- sorgungsverantwortung}

Insgesamt zeigt sich damit, daß das $\mathrm{KrW}$-/ AbfG kaum über Instrumente verfügt, eine saubere Unterscheidung zwischen der privaten Verwertungsverantwortlichkeit und der öffentlich-rechtlichen Beseitigungshoheit durchzuführen. Letztlich ist es nicht geeignet, Abfallströme im Sinne eines Stoffstrommanagements zu steuern. Vielmehr bleiben die vielfältigen Abgrenzungskriterien zwischen dem Begriff des Abfalls zur Verwertung und dem des Abfalls zur Beseitigung letztlich wirkungslos. Die Steuerung erfolgt nahezu ausschließlich über den Preis. Stoffrelevante Bestimmungen des $\mathrm{KrW}-/ \mathrm{AbfG}$ finden in der Praxis bislang keine Anwendung; sie sind nahezu wirkungslos. Auch sie stellen ein eher symbolisches Regelungsgerüst dar. Letztlich fehlt es damit auch an einer sauberen und in der Praxis umzusetzenden Abgrenzung zwischen öffentlicher und privater Verantwortlichkeit in der Abfallwirtschaft. Regelungsinstrument ist derzeit allein der Preis und die Verwertungsoption des Abfallbesitzers. Dieser entscheidet nach Kostengesichtspunkten, wie er mit dem Abfall verfährt. Mit einer ökologischen, stoffstromorientierten Kreislaufwirtschaft hat dies nur sehr wenig zu tun.

\section{Schlußbemerkung}

Bei näherem Hinsehen zeigt sich, daß die Zielsetzungen des Kreislaufwirtschafts- und Abfallgesctzes, einer Vermeidung von Abfällen oberste Priorität zu geben, dem Produzenten eine Verantwortung für sein Produkt zuzuweisen und eine neue Verantwortungshierarchie mit dem Vorrang private. Entsorgung zu schaffen, durch das $\mathrm{KrW}$-/AbfG kaum steuerungsfähig sind. Der Gesetzgeber hat es beim Verwertungsvorrang sowie der Regelung über die Produktverantwortung weitgehend bei Bestimmungen mit Appellcharakter belassen und dem Verordnungsgeber im einzelnen auferlegt, Detailregelungen für einzelne Sachbereiche zu treffen. Solche Regelungen

69 In diesem Sinne Hosel/von Lersner/Wendenburg, KrW-/AbfG, \$s Rdnr. 34; Kunig, in: Kunig/Paetorv/ Versteyl, KrW-/AbfG (Fn. 14), \$s Rdnr. 17; àhlich auch Frenz, KrW-/AbfG (Fn. 10), \$s Rdnr. 40: Ernffrnung der Moglichkeit, von der Rangfolge Verwertung/Beseitigung abzuweichen. A. A. Klock, ZLK $1995,117,119$ 
gibt es zwar, wie die VerpackV, die AltautoV und die BattV zeigen. An Instrumenten zur Umsetzung der Vermeidungsverpflichtung fehlt es jedoch auch in diesen Verordnungen weitestgehend. Auch was dic Produktverantwortung angeht, sind sie in vielen Bcreichen lückenhaft. Eine Gesamtkonzeption der Vermeidung von Abfällen und der Produktverantwortung fehlt weitgehend. Gerade die diesbezüglichen Bestimmungen des $\mathrm{KrW}-/ \mathrm{AbfG}$ haben eher symbolischen Charakter. Ähnliches gilt für die neue Verantwortungszuweisung an private Entsorgungsträger, die eine Abkehr von dem bisherigen Entsorgungsmonopol der öffentlichen Hand bringen soll. Rechtlich hat sich durch diese neuen Bestimmungen sehr wenig geändert, da die Verantwortungszuweisung für die Entsorgung an Private durch die Überlassungspflichten letztlich wieder aufgehoben und die öffentlich-rechtliche Entsorgungsverantwortung quasi durch die Hintertür wieder eingeführt wird. Die Abgrenzung von Entsorgungszuständigkeiten zwischen Privaten und der öffentlichen Hand erfolgt nahezu ausschließlich über den Preis. Der Abfallerzeuger und -besitzer hat es in der Hand, zu entscheiden, ob scine Abfälle verwertet oder beseitigt werden. Ist die Verwertung für ihn - was häufig der Fall ist - kostengünstiger, wird er diese, nicht aber die Beseitigung wählen. Verläßliche Abgrenzungskriterien zwischen beiden Entsorgungsarten und damit für die Entscheidung zwischen privater und öffentlicher Entsorgung fehlen weitgehend. Die diesbezüglichen Regelungen haben entweder, wie das Postulat nach Hochwertigkeit der Verwertung, nur appellativen Charakter oder haben sich - wie das Hauptzweckkriterium als Merkmal zur Scheidung zwischen Abfällen zur Verwertung und Beseitigung oder die Forderung nach Schadlosigkeit der Verwertung oder nach Wahl der umwcltverträglicheren Entsorgungsart - in der Praxis bislang nicht als brauchbare Abgrenzungskriterien erwiesen. Auch insoweit verbleibt cs im $\mathrm{KrW}-/ \mathrm{AbfG}$ weitgehend bei symbolhaften Regelungen. Eine Steuerungsfunktion kommt den genannten Bestimmungen jedenfalls nicht zu. Die Abgrenzung zwischen privater und öffentlicher Verantwortung in der Abfallentsorgung ist damit zwar nicht ausschließlich symbolisch. Sie hat jedoch sehr starke symbolische Einschläge. Im Ergebnis bleibt festzustellen, daß es an einer ökologischen, stoffstromorientierten Steuerung im Abfallrecht mangelt. Der symbolische Charaktcr der neuen, auf eine Kreislaufwirtschaft orientierten Regelungen ist unverkennbar. 